ACADEMIA ROMÂNĂ

Revue Roumaine de Chimie

http://web.icf.ro/rrch/
Rev. Roum. Chim.,

2020, 65(6), 517-528

DOI: 10.33224/rrch.2020.65.6.01

\title{
NEW TOCOPHEROL DERIVATIVES FOR FUNCTIONALIZATION OF AMINO OR CARBOXYL GROUPS WITH LIPID ANCHORS
}

\author{
Ralph SPRINGER $^{\mathrm{a}}$ and Jürgen LIEBSCHER ${ }^{\mathrm{a}, \mathrm{b},{ }^{*}}$ \\ ${ }^{a}$ Institute of Chemistry, Humboldt-University Berlin, Brook-Taylor-Str. 2, 12489 Berlin, Germany. \\ ${ }^{\mathrm{b}}$ National Institute for Research and Development of Isotopes and Molecular Technologies (INCDTIM), Strada Donat 67-103, \\ 400293 Cluj-Napoca, Roumania
}

Lipid anchors play an important biological role in natural proteins in particular in lipid membrane anchoring. This principle was extended to nonnatural nucleic acids, peptide nucleic acids (PNA) and peptides. In order to provide<smiles>C#CCNc1nc(NCCCCC(=O)O)nc(NCCCCC(=O)O)n1</smiles><smiles>O=C(O)Cc1cn(CC(Cn2nnc(CC(=O)O)n2)OCC#Cc2cn(CC(=O)O)c(=O)[nH]c2=O)nn1</smiles>
new lipophilic anchors for the introduction into peptide nucleic acids (PNA) or peptides, a number of new $\alpha$-tocopherol derivatives were synthesized containing carboxylic acids, amino groups or alkyne groups as linking sites. Amongst them are compounds with one or with two tocopherol units. Sonogashira reaction turned out to be a useful tool in these approaches. The products were characterized by NMR-spectroscopy and MS. An unusual phenomenon was found in 2-propargylamino-4,6-difluorotriazine that exhibits two different chemical shifts in the ${ }^{19} \mathrm{~F}-\mathrm{NMR}$ spectrum for the two fluoro atoms.

\section{INTRODUCTION}

Lipid anchors play an important role in biochemistry, e.g. in fixing proteins to lipid membranes. In nature, isoprenoides or fatty acids often serve as lipid anchors. Unnatural anchoring of nucleic acid (DNA or RNA) sequences was achieved by the introduction of lipid anchors like fatty acids, long chain alkyl groups or isoprenoides, $\alpha$-tocopherol or cholesterol. ${ }^{1-3}$ Such chimers have found many interesting applications not only in the lipid membrane field. The strategy of introduction of lipid anchors was also applied to peptide nucleic acids (PNA) $1 .^{4-8}$

By proper choice of the type of anchor as well as the position of its fixation, domain-specific anchoring in lipid-ordered and lipid-disordered domains of vesicles was achieved. ${ }^{1,4,5}$ In cases of PNA, lipid anchors were only covalently fixed at the $\mathrm{N}$-terminus of PNAs. We report here the synthesis of new $\alpha$ tocopherol derivatives that can be linked as termini to either ends of peptides or PNAs or in the middle of a PNA as lipophilic nucleobase. These derivatives contain either carboxyl or amino groups.

\footnotetext{
*Corresponding author: liebscher@chemie.hu-berlin.de
} 


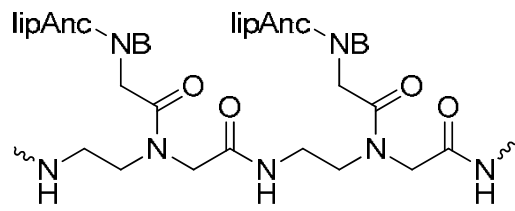

1

\section{RESULTS AND DISCUSSION}

In order to obtain derivatives of $\alpha$-tocopherol with carboxyl groups, we aimed to compounds containing uracil as a recognition function in nucleic acid chemistry. 5-Iodouracil 2 underwent Sonogashira reaction with $O$-propargyltocopherol $\mathbf{3}^{9}$ resulting in the coupling product $\mathbf{4}$ in $77 \%$ yield (Scheme 1). Subsequent N-alkylation with $t$-butyl bromoacetate took predominately place in position 1 affording 5. However, some dialkylation product 6 was observed in addition. Surprisingly, we encountered some decomposition problems in cleaving the tert-butyl ester moiety of $\mathbf{5}$. Therefore we chose another way towards the synthesis of the
lipAnc = lipophilic anchoring group

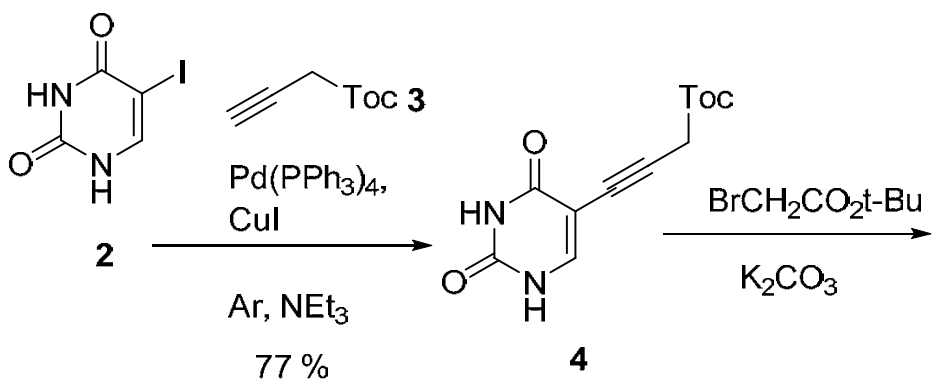<smiles>CCCCOC(=O)CCn1cc(C#CCC(=O)OCc2ccccc2)c(=O)n(CC(=O)OCC(C)(C)C)c1=O</smiles>

5 corresponding acid 8 by reversing the sequence of Sonogashira reaction and N-alkylation. Reaction of 5 -iodouracil with bromoacetic acid in the presence of potassium carbonate gave $52 \%$ of the (5-iodouracilyl) acetic acid 7. Prolongation of the reaction time did not increase the yield. The following Sonogashira reaction of 7 with the propargylated $\alpha$-tocopherol $\mathbf{3}$ performed satisfactorily (75\% yield) leading to the carboxylic acid 8 directly, i.e. without a deprotection step. This carboxylic acid $\mathbf{8}$ represents a candidate for the introduction of the tocopherol-uracil conjugate as a side chain in PNAs or at the N-terminus of peptides and PNAs by $\mathrm{N}$-acylation.

$$
\mid \begin{gathered}
\mathrm{KOH} / \mathrm{H}_{2} \mathrm{O} \\
40{ }^{\circ} \mathrm{C} \\
\mathrm{BrCH}_{2} \mathrm{CO}_{2} \mathrm{H} \\
52 \%
\end{gathered}
$$

$68 \%$<smiles>O=C(O)Cn1cc(I)c(=O)[nH]c1=O</smiles>

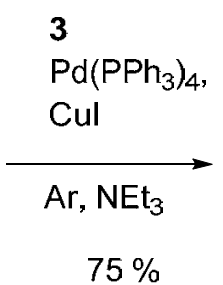<smiles>O=C(O)CC#Cc1cn(CC(=O)O)c(=O)[nH]c1=O</smiles> 

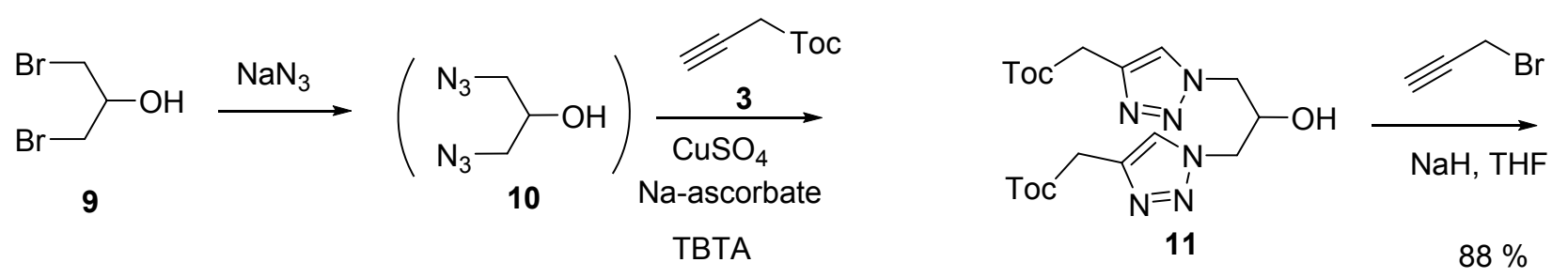

$55 \%$

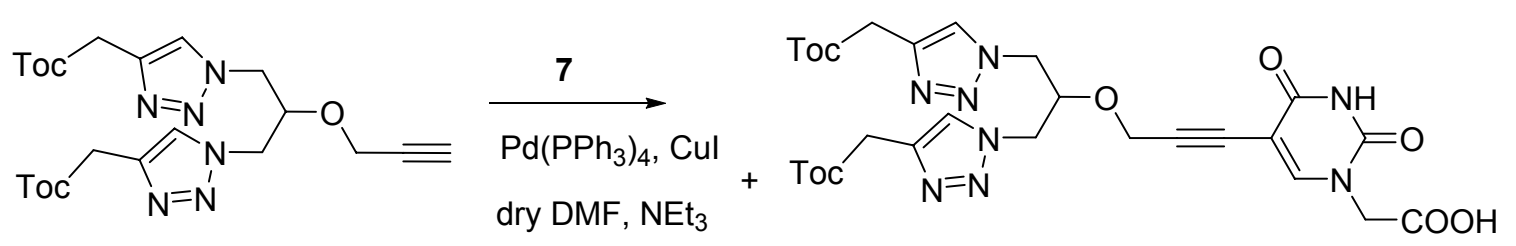

12

13

Scheme 2 - Synthesis of uracil-1-yl acetic acid 13 containing two $\alpha$-tocopherol units.

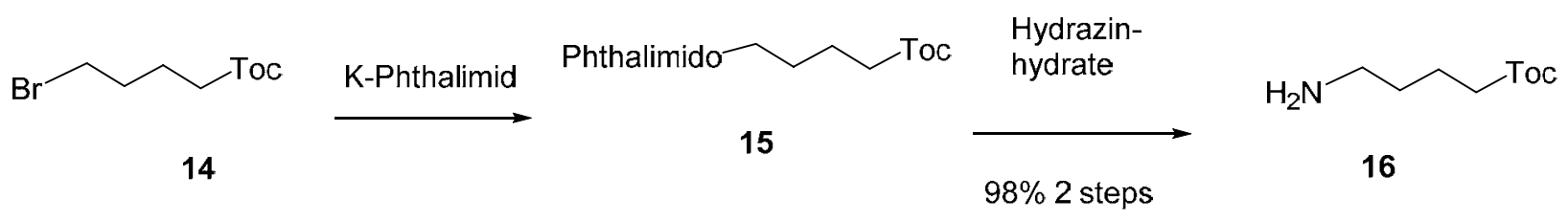

Scheme 3 - Synthesis of $O$-(4-aminobutyl)-tocopherol 16.

It can be surmised that a system containing two $\alpha$-tocopherol units could eventually provide stronger anchoring properties in lipid membranes. Consequently, we tried to synthesize such an assembly. Again we chose a conjugate with uracil as target making use of the experiences with the synthesis of tocopherol-uracil-carboxylic acid 8 . Now, the two tocopherol units were linked to an isopropyl alcohol unit (Scheme 2). 2-Hydroxy-1,3dibromopropane 9 was transformed into the diazide 10 by reaction with sodium azide following a modified reported procedure omitting the isolation of potentially explosive diazide. ${ }^{10} \mathbf{1 0}$ underwent in situ $\mathrm{CuAAC}$ click reaction with $O$ propargyltocopherol 3 resulting in ditriazole 11. $O$ Propargylation was performed with propargyl bromide in the presence of sodium hydride giving access to the ether $\mathbf{1 2}$ in $88 \%$ yield. The Sonogashira reaction of the propargyl ether $\mathbf{1 2}$ with the iodouracilylacetic acid 7 to the target product 13 occurred as found by HPLC-MS. However, the isolation of the product in preparative yields turned out to be difficult.

We further envisaged synthesizing tocopherol anchors containing other tethers than carboxyl. A primary amino group was introduced into $\alpha$ tocopherol in a straight forward 3-step synthesis. Alkylation of tocopherol with 1,4-dibromobutane resulted in the bromobutyl ether $\mathbf{1 4}$ that further underwent Gabriel synthesis by first reaction with phthalimide (formation of 15) and subsequent hydrazinolysis. $98 \%$ Yield of the $O$-(4aminobutyl)-tocopherol $\mathbf{1 6}$ was obtained over the last two steps (Scheme 3). This product $\mathbf{1 6}$ is suitable for the introduction of tocopherol anchors at the C-terminus of peptides or PNAs.

On the other hand, 16 can also be used to construct a di-tocopheryl anchor useful as alkyne component in Sonogashira reactions. 2,4,6Trifluoro-1,3,5-triazine (cyanuric fluoride) $\mathbf{1 7}$ is known to react selectively with amines by stepwise substitution of the fluoro atoms. In the first step, one fluoride was replaced by propargyl amine affording 18 (Scheme 4). The remaining two fluoro atoms were substituted by the $O$-(4-aminobutyl)tocopherol 16 leading to the target molecule 19 in $92 \%$ yield. The product is an interesting candidate that could be used for the introduction of a double lipid anchor into 5-iodouracil by Sonogashira reaction.

As an unexpected phenomenon, two different sets of signals were observed for the two fluoro atoms in the ${ }^{19} \mathrm{~F}-\mathrm{NMR}$ spectrum of the monosubstituted product 18, although the molecule is symmetric. X-Ray crystal analysis of $\mathbf{1 8}$ confirmed the structure but also showed 
intermolecular H-F bridging with a H-F distance of $2.8 \AA$ of one of the fluoro atoms, rendering the two fluoro atoms non-equivalent. It is likely that the situation is similar in solution and thus can explain the non-equivalence of the two fluoro atoms in the ${ }^{19}$ F-NMR-spectum.

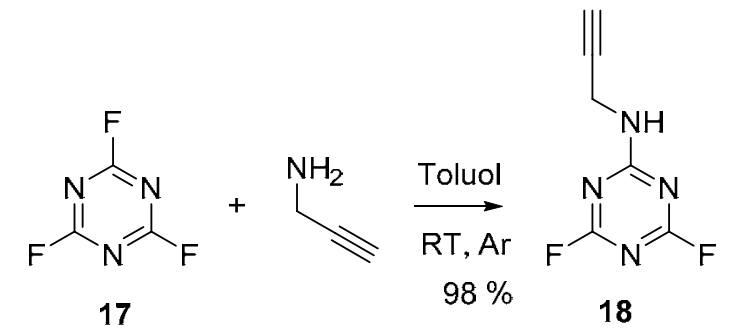<smiles></smiles>

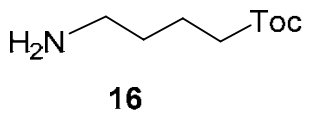

(92\%)<smiles>C#CCNc1nc(NCCCCCC(=O)O)nc(NCCCCC(=O)O)n1</smiles>

Scheme 4 - Synthesis of 2-propargylamino-1,3,5-triazine 19 containing two tocopherol units in positions 4 and 6.

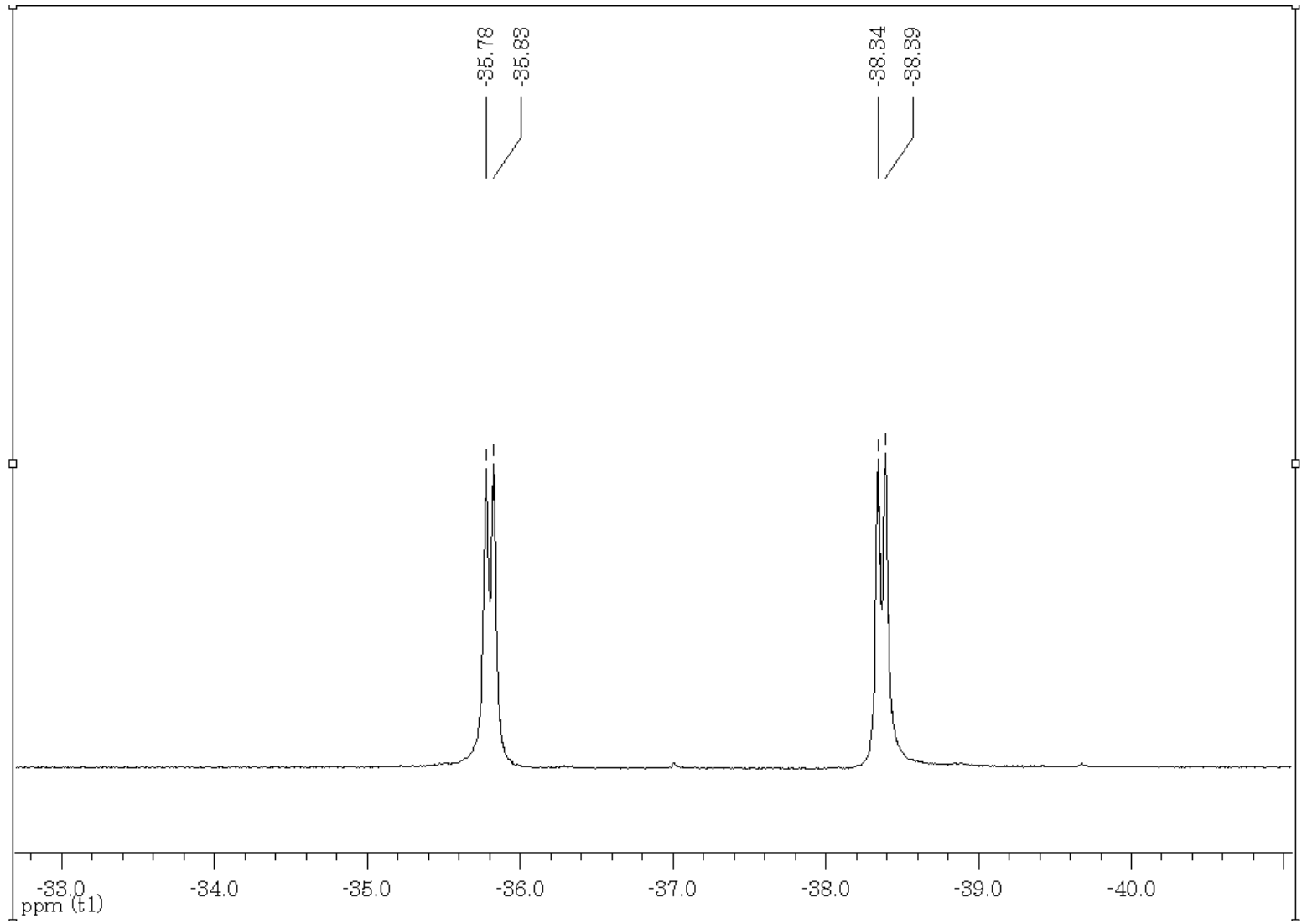

Fig. $1-{ }^{19}$ F-NMR spectrum of 2-propargylamino-4,6-difluorotriazine 18. 

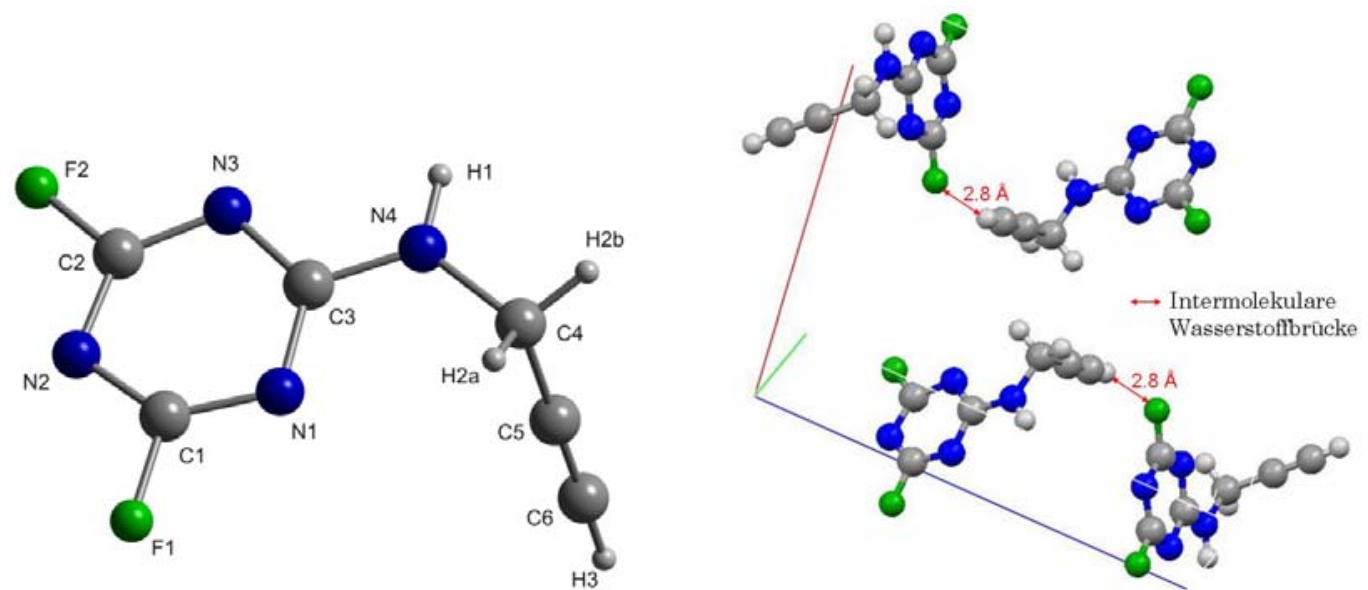

Fig. 2 - X-ray crystal analysis of 2-propargylamine-4,6-difluorotriazine 18.

\section{EXPERIMENTAL}

\section{Instruments}

NMR-Spectra were recorded at a AV 300 or AV 400 spectrometer from Bruker. Internal standard: tetramethylsilane. EI-HRMS spectra were obtained by a spectrometer MAT 711 from Varian and ESI-HRMS spectra by a Finnigan LTQ FTmasspectrometer, Thermo Electron. Elemental analysis were determined with an EuroEA 3000 instrument from HEKAtech. XRay crystal analysis was performed with IPDS- or STADI-4 diffractometer from STOE \& Cie.

\section{Materials}

Chemicals were purchased from Acros, Sigma Aldrich, IrisBiotech, NovoBiotech and Merck. The were used without further purification if not otherwise mentioned. $O$ Propargyltocopherol was obtained by a literature procedure. ${ }^{9}$

\section{O-[1-(uracil-5-yl)-prop-1-yne-3-yl]-tocopherol) (4) (adapted from ref. $\left.{ }^{9}\right)$ \\ $\mathrm{Pd}\left(\mathrm{PPh}_{3}\right)_{4} \quad(0.20 \mathrm{~g}, \quad 0.18 \mathrm{mmol})$ and $\mathrm{CuI} \quad(0.11 \mathrm{~g}$,} $0.58 \mathrm{mmol})$ were added to a solution of iodouracil $2(1.19 \mathrm{~g}$, $5.00 \mathrm{mmol})$ in dry DMF $(10 \mathrm{~mL})$ under argon. A solution of (O-propargyl)-tocopherol 3 (9.38 g, $20.00 \mathrm{mmol})$ and triethyl amine $(0.73 \mathrm{~mL}, 8.70 \mathrm{mmol})$ were added dropwise. After stirring overnight the solvent was removed under vacuum and the remainder purified by column chromatography $(1000 \mathrm{~g}$ silica, cyclohexane/ethyl acetate $1: 1)$ yielding product $4(2.23$ g, $77 \%$ ) as colorless yellowish solid. m. p. $174-176^{\circ} \mathrm{C}$. Anal. calcd for $\mathrm{C}_{36} \mathrm{H}_{55} \mathrm{~N}_{2} \mathrm{O}_{4}$ : C 74.70, H 9.40, N 4.84. Found: $\mathrm{C}$ 74.09, $\mathrm{H} 9.58, \mathrm{~N}$ 4.65. HRMS (ESI) $m / z\left(\mathrm{M}+\mathrm{H}^{+}\right) \mathrm{C}_{36} \mathrm{H}_{55} \mathrm{~N}_{2} \mathrm{O}_{4}$ calcd. 579.4162, found: 579.4185. ${ }^{1} \mathrm{H}-\mathrm{NMR}(300 \mathrm{MHz}$, $\left.\mathrm{CDCl}_{3}\right): \delta(\mathrm{ppm})=10.35(\mathrm{~d}, J=5.1 \mathrm{~Hz}, 1 \mathrm{H}(\mathrm{NH} 1)), 10.02(\mathrm{~s}$ $1 \mathrm{H}(\mathrm{NH} 3)), 7.61(\mathrm{~d}, J=6.0 \mathrm{~Hz}, 1 \mathrm{H}(\mathrm{CH} 6)), 4.54(\mathrm{~s}, 2 \mathrm{H}(\mathrm{C} \equiv \mathrm{C}-$ $\left.\left.\mathrm{CH}_{2}-\mathrm{O}\right)\right), 2.56\left(\mathrm{t}, J=6.6 \mathrm{~Hz}, 2 \mathrm{H}\left(\mathrm{O}-\mathrm{C}_{\mathrm{q}}-\mathrm{CH}_{2}-\mathrm{CH}_{2}-\mathrm{C}_{\mathrm{q}}\right)\right), 2.20(\mathrm{~s}$, $\left.3 \mathrm{H}\left(\mathrm{C}_{\mathrm{ar} .}-\mathrm{CH}_{3}\right)\right), 2.16\left(\mathrm{~s}, 3 \mathrm{H}\left(\mathrm{C}_{\mathrm{ar} .}-\mathrm{CH}_{3}\right)\right), 2.07\left(\mathrm{~s}, 3 \mathrm{H}\left(\mathrm{C}_{\mathrm{ar} .}\right.\right.$. $\left.\mathrm{CH}_{3}\right)$ ), 1.71-1.82 (m, 2H (O- $\left.\left.\mathrm{C}_{\mathrm{q}}-\mathrm{CH}_{2}-\mathrm{C}_{2}-\mathrm{C}_{\mathrm{q}}\right)\right), 1.07-1.59(\mathrm{~m}$, $\left.24 \mathrm{H}\left(3 \times \mathrm{CH}, 9 \times \mathrm{CH}_{2}, 1 \times \mathrm{CH}_{3}\right)\right), 0.80-0.89(\mathrm{~m}, 12 \mathrm{H}(4 \times \mathrm{CH}-$ $\left.\left.\mathrm{C}_{3}\right)\right) .{ }^{13} \mathrm{C}-\mathrm{NMR}\left(75 \mathrm{MHz}, \mathrm{CDCl}_{3}\right): \delta(\mathrm{ppm})=163.2(\mathrm{O}=\mathrm{C} 4)$, $151.7(\mathrm{O}=\mathrm{C} 2), 148.2\left(\mathrm{O}-\mathrm{C}_{\mathrm{ar}}\right), 147.8\left(\mathrm{O}-\mathrm{C}_{\mathrm{ar}}\right), 144.9(\mathrm{CH} 6)$, $127.9\left(\mathrm{C}_{\mathrm{ar}}\right), 126.0\left(\mathrm{C}_{\mathrm{ar}}\right), 123.0\left(\mathrm{C}_{\mathrm{ar}}\right), 117.6\left(\mathrm{C}_{\mathrm{ar} .}\right), 99.3\left(\mathrm{C}_{\mathrm{ar}} 5\right)$, $90.1\left(-\mathrm{C} \equiv \mathrm{C}-\mathrm{CH}_{2}\right), 76.8\left(-\mathrm{C} \equiv \mathrm{C}-\mathrm{CH}_{2}\right), 74.9\left(\mathrm{O}-\mathrm{C}_{\mathrm{q}}-\left(\mathrm{CH}_{2}\right)_{2} \mathrm{CH}_{3}\right)$, $61.2\left(\equiv \mathrm{C}-\mathrm{CH}_{2}-\mathrm{O}\right), 40.1\left(\mathrm{CH}_{2}\right), 39.3\left(\mathrm{CH}_{2}\right), 37.4\left(\mathrm{CH}_{2}\right), 37.4$ $\left(\mathrm{CH}_{2}\right), 37.2\left(\mathrm{CH}_{2}\right), 32.7\left(\mathrm{CH} / \mathrm{CH}_{3}\right), 32.7\left(\mathrm{CH} / \mathrm{CH}_{3}\right), 31.2\left(\mathrm{C}_{\text {ar. }}\right.$ -
$\left.\mathrm{CH}_{2}-\mathrm{CH}_{2}\right), 31.1\left(\mathrm{CH}_{2}\right), 27.9\left(\mathrm{CH} / \mathrm{CH}_{3}\right), 24.8\left(\mathrm{CH}_{2}\right), 24.4$ $\left(\mathrm{CH}_{2}\right), 23.7\left(\mathrm{CH} / \mathrm{CH}_{3}\right), 22.7\left(\mathrm{CH} / \mathrm{CH}_{3}\right), 22.6\left(\mathrm{CH} / \mathrm{CH}_{3}\right), 21.0$ $\left(\mathrm{CH}_{2}\right), 20.6\left(\mathrm{CH}_{2}-\mathrm{CH}_{2}-\mathrm{C}_{\mathrm{q}}\right), 19.7\left(\mathrm{CH} / \mathrm{CH}_{3}\right), 19.6\left(\mathrm{CH} / \mathrm{CH}_{3}\right)$, $13.1\left(\mathrm{C}_{\mathrm{ar}}-\underline{\mathrm{C}} \mathrm{CH}_{3}\right), 12.2\left(\mathrm{C}_{\mathrm{ar}}-\underline{\mathrm{C}} \mathrm{H}_{3}\right), 11.8\left(\mathrm{C}_{\mathrm{ar}}-\underline{\mathrm{C}} \mathrm{H}_{3}\right)$.

\section{(Tocopher-6-yloxy)-prop-1-inyl)uracilyl)-substituted tert- butyl acetate (5) and dialkylation product (6)}

The reaction was performed under argon with exclusion of light. tert-Butyl bromo acetate $(0.30 \mathrm{~mL}, 2.00 \mathrm{mmol})$ in dry DMF $(1 \mathrm{ml})$ was added dropwise at $0{ }^{\circ} \mathrm{C}$ to a suspension of lipidated 5-(tocopher-6-yloxy)-prop-1-inyl)uracil 4 (1.16 g, $2.00 \mathrm{mmol})$ and $\mathrm{K}_{2} \mathrm{CO}_{3}(0.30 \mathrm{~g}, 2.20 \mathrm{mmol})$ in dry DMF $(8$ $\mathrm{ml}$ ). The mixture was allowed to warm up to room temperature (rt) and was stirred overnight. Solids were filtered off and washed with DMF several times. Combined filtrates were concentrated under vacuum and remainders of the solvents were removed by aceotropic distillation with toluene. The crude product was dissolved in ethyl acetate (EtOAc) $(10$ $\mathrm{mL})$ and washed with water $(8 \mathrm{~mL})$ three times and once with brine. The aqueous washing phases were re-extracted with EtOAc $(10 \mathrm{~mL})$ three times and the combined organic phases were dried over $\mathrm{Na}_{2} \mathrm{SO}_{4}$. After removing the solvent under vacuum the remainder was purified by column chromatography ( $250 \mathrm{~g}$ silica, cyclohexane/ethyl acetate $7: 3$ ) yielding $0.94 \mathrm{~g}(68 \%)$ of 5 as yellowish solid and $0.31 \mathrm{~g} \mathrm{(15}$ $\%)$ of the dialkylation product $\mathbf{6}$ as brownish oil.

5: $\mathrm{R}_{\mathrm{f}}=0.29$ (cyclohexane/EtOAc $7: 3$ ). m. p. $102-104{ }^{\circ} \mathrm{C}$. HRMS (ESI) $m / z\left(\mathrm{M}+\mathrm{H}^{+}\right) \mathrm{C}_{42} \mathrm{H}_{65} \mathrm{~N}_{2} \mathrm{O}_{6}$ calcd. 693.4843, found 693.4858. ${ }^{1} \mathrm{H}-\mathrm{NMR}\left(300 \mathrm{MHz}, \mathrm{CDCl}_{3}\right): \delta(\mathrm{ppm})=8.58(\mathrm{~s}, 1 \mathrm{H}$ (NH3)), 7.41 (s, 1H (CH6)), $4.55\left(\mathrm{~s}, 2 \mathrm{H}\left(\mathrm{C} \equiv \mathrm{C}_{-} \mathrm{CH}_{2}-\mathrm{O}\right)\right), 4.37$ $\left(\mathrm{s}, 2 \mathrm{H}\left(\mathrm{N} 1-\mathrm{CH}_{2}-\mathrm{CO}_{2}\right)\right), 2.57\left(\mathrm{t}, J=6.7 \mathrm{~Hz}, 2 \mathrm{H}\left(\mathrm{O}-\mathrm{C}_{\mathrm{q}}-\mathrm{CH}_{2}-\right.\right.$ $\left.\left.\mathrm{CH}_{2}-\mathrm{C}_{\mathrm{q}}\right)\right), 2.21\left(\mathrm{~s}, 3 \mathrm{H}\left(\mathrm{C}_{\mathrm{ar}}-\mathrm{CH}_{3}\right)\right), 2.17\left(\mathrm{~s}, 3 \mathrm{H}\left(\mathrm{C}_{\mathrm{ar}}-\mathrm{CH}_{3}\right)\right), 2.07$ (s, $\left.3 \mathrm{H}\left(\mathrm{C}_{\mathrm{ar}}-\mathrm{CH}_{3}\right)\right), 1.71-1.85\left(\mathrm{~m}, 2 \mathrm{H},\left(\mathrm{O}-\mathrm{C}_{\mathrm{q}}-\mathrm{CH}_{2}-\mathrm{CH}_{2}-\mathrm{C}_{\mathrm{q}}\right)\right.$ ), $1.49\left(\mathrm{~s}, 9 \mathrm{H}\left(3 \times \mathrm{O}-\mathrm{C}_{\mathrm{q}}-\mathrm{CH}_{3}\right)\right), 0.99-1.60(\mathrm{~m}, 24 \mathrm{H}(3 \times \mathrm{CH}$, $\left.\left.9 \times \mathrm{CH}_{2}, 1 \times \mathrm{CH}_{3}\right)\right), 0.80-0.89\left(\mathrm{~m}, 12 \mathrm{H}\left(4 \times \mathrm{CH}-\mathrm{CH}_{3}\right)\right) .{ }^{13} \mathrm{C}-\mathrm{NMR}$ $\left(75 \mathrm{MHz}, \mathrm{CDCl}_{3}\right): \delta(\mathrm{ppm})=165.9\left(-\mathrm{CO}_{2}\right), 161.5(\mathrm{O}=\mathrm{C} 4)$, $149.7(\mathrm{O}=\mathrm{C} 2), 148.2\left(\mathrm{O}-\underline{C}_{\mathrm{ar}}\right), 147.7\left(\mathrm{O}-\underline{\mathrm{C}}_{\mathrm{ar}}\right), 147.7(\underline{\mathrm{CH}})$, $128.0\left(\mathrm{C}_{\mathrm{ar} .}\right), 126.1\left(\mathrm{C}_{\mathrm{ar} .}\right), 122.9\left(\mathrm{C}_{\mathrm{ar} .}\right), 117.6\left(\mathrm{C}_{\mathrm{ar}}\right), 99.8\left(\mathrm{C}_{\mathrm{ar}}\right)$ ), $90.1\left(-\mathrm{C} \equiv \mathrm{C}-\mathrm{CH}_{2}\right), 83.8\left(\mathrm{O}-\mathrm{C}_{\mathrm{q}}-\left(\mathrm{CH}_{3}\right)_{3}\right), 76.7\left(-\mathrm{C} \equiv \mathrm{C}-\mathrm{CH}_{2}\right), 74.9$ $\left(\mathrm{O}-\mathrm{C}_{\mathrm{q}}-\left(\mathrm{CH}_{2}\right)_{2} \mathrm{CH}_{3}\right), 61.1\left(\mathrm{C} \equiv \mathrm{C}-\mathrm{CH}_{2}\right), 49.6\left(\mathrm{~N} 1-\mathrm{CH}_{2}-\mathrm{CO}_{2}\right), 40.1$ $\left(\mathrm{CH}_{2}\right), 39.3\left(\mathrm{CH}_{2}\right), 37.5\left(\mathrm{CH}_{2}\right), 37.4\left(\mathrm{CH}_{2}\right), 37.2\left(\mathrm{CH}_{2}\right), 32.7$ $\left(\mathrm{CH} / \mathrm{CH}_{3}\right), 32.7\left(\mathrm{CH} / \mathrm{CH}_{3}\right), 31.2\left(\mathrm{C}_{\mathrm{ar}}-\mathrm{CH}_{2}-\mathrm{CH}_{2}\right), 31.1\left(\mathrm{CH}_{2}\right)$, $28.0\left(\mathrm{O}-\mathrm{C}_{\mathrm{q}}-\mathrm{CH}_{3}\right), 24.8\left(\mathrm{CH}_{2}\right), 24.4\left(\mathrm{CH}_{2}\right), 23.8\left(\mathrm{CH} / \mathrm{CH}_{3}\right)$, $22.7\left(\mathrm{CH} / \mathrm{CH}_{3}\right), 22.6\left(\mathrm{CH} / \mathrm{CH}_{3}\right), 21.0\left(\mathrm{CH}_{2}\right), 20.6\left(\mathrm{CH}_{2}-\mathrm{CH}_{2}-\right.$ $\left.\mathrm{C}_{\mathrm{q}}\right), 19.7\left(\mathrm{CH} / \mathrm{CH}_{3}\right), 19.6\left(\mathrm{CH} / \mathrm{CH}_{3}\right), 19.6\left(\mathrm{CH} / \mathrm{CH}_{3}\right), 13.1$ $\left(\mathrm{C}_{\mathrm{ar} .}-{ }_{\mathrm{C}} \mathrm{H}_{3}\right), 12.2\left(\mathrm{C}_{\mathrm{ar} .}-{ }_{\mathrm{C}} \mathrm{H}_{3}\right), 11.8\left(\mathrm{C}_{\mathrm{ar} .}-\underline{\mathrm{C}} \mathrm{H}_{3}\right)$. 


\section{Dialkylation product 6}

$\mathrm{R}_{\mathrm{f}}=0.59$ (cyclohexane/EtOAc $7: 3$ ). Anal. calcd. for $\mathrm{C}_{48} \mathrm{H}_{74} \mathrm{~N}_{2}$ : C 71.43, H 9.24, N 3.47, found C 71.59, H 9.38, N 3.25. ${ }^{1} \mathrm{H}-\mathrm{NMR}\left(300 \mathrm{MHz}, \mathrm{CDCl}_{3}\right): \delta(\mathrm{ppm})=7.43(\mathrm{~s}, 1 \mathrm{H}$ (CH6)), $4.60\left(\mathrm{~s}, 2 \mathrm{H}\left(\mathrm{N} 1-\mathrm{CH}_{2}-\mathrm{CO}_{2}\right)\right)^{*}, 4.54\left(\mathrm{~s}, 2 \mathrm{H}\left(\mathrm{C} \equiv \mathrm{C}_{-}-\mathrm{CH}_{2}-\right.\right.$ $\mathrm{O})), 4.37\left(\mathrm{~s}, 2 \mathrm{H}\left(\mathrm{N} 3-\mathrm{CH}_{2}-\mathrm{CO}_{2}\right)\right)^{*}, 2.57\left(\mathrm{t}, J=6.7,2 \mathrm{H}\left(\mathrm{O}-\mathrm{C}_{\mathrm{q}}-\right.\right.$ $\left.\left.\mathrm{CH}_{2}-\mathrm{CH}_{2}-\mathrm{C}_{\mathrm{q}}\right)\right), 2.21\left(\mathrm{~s}, 3 \mathrm{H}\left(\mathrm{C}_{\mathrm{ar}}-\mathrm{CH}_{3}\right)\right), 2.17\left(\mathrm{~s}, 3 \mathrm{H}\left(\mathrm{C}_{\mathrm{ar}}-\right.\right.$ $\left.\left.\mathrm{CH}_{3}\right)\right), 2.07\left(\mathrm{~s}, 3 \mathrm{H}\left(\mathrm{C}_{\mathrm{ar} .}-\mathrm{CH}_{3}\right)\right), 1.68-1.85\left(\mathrm{~m}, 2 \mathrm{H},\left(\mathrm{O}-\mathrm{C}_{\mathrm{q}}-\mathrm{CH}_{2}-\right.\right.$ $\left.\left.\mathrm{CH}_{2}-\mathrm{C}_{\mathrm{q}}\right)\right), 1.01-1.59\left(\mathrm{~m}, 24 \mathrm{H}\left(3 \times \mathrm{CH}, 9 \times \mathrm{CH}_{2}, 1 \times \mathrm{CH}_{3}\right)\right), 1.48$ $\left(\mathrm{s}, 9 \mathrm{H}\left(3 \times \mathrm{O}-\mathrm{C}_{\mathrm{q}}-\mathrm{CH}_{3}\right)\right), 1.46\left(\mathrm{~s}, 9 \mathrm{H}\left(3 \times \mathrm{O}-\mathrm{C}_{\mathrm{q}}-\mathrm{CH}_{3}\right)\right), 0.81-0.91$ (m, $\left.12 \mathrm{H}\left(4 \times \mathrm{CH}_{-} \mathrm{CH}_{3}\right)\right) .{ }^{13} \mathrm{C}-\mathrm{NMR}\left(75 \mathrm{MHz}, \mathrm{CDCl}_{3}\right): \delta(\mathrm{ppm})$ $=166.2\left(\mathrm{~N} 3-\mathrm{CH}_{2}-\mathrm{CO}_{2}\right), 166.0\left(\mathrm{~N} 1-\mathrm{CH}_{2}-\mathrm{CO}_{2}\right), 160.9(\mathrm{O}=\mathrm{C} 4)$, $150.2(\mathrm{O}=\mathrm{C} 2), 148.2\left(\mathrm{O}-\underline{\mathrm{C}}_{\mathrm{ar} .}\right), 147.8\left(\mathrm{O}-\underline{\mathrm{C}}_{\mathrm{ar} .}\right), 146.5(\mathrm{CH} 6)$, $\left.128.0\left(\mathrm{C}_{\mathrm{ar}}\right), 126.1\left(\mathrm{C}_{\mathrm{ar}}\right), 122.9\left(\mathrm{C}_{\mathrm{ar}}\right), 117.5\left(\mathrm{C}_{\mathrm{ar}}\right), 98.7\left(\mathrm{C}_{\mathrm{ar}}\right)\right)$, $89.5\left(-\mathrm{C} \equiv \mathrm{C}-\mathrm{CH}_{2}\right), 83.4\left(\mathrm{O}-\underline{\mathrm{C}}_{\mathrm{q}}-\left(\mathrm{CH}_{3}\right)_{3}\right), 82.3\left(\mathrm{O}-\underline{\mathrm{C}}_{\mathrm{q}}-\left(\mathrm{CH}_{3}\right)_{3}\right)$, $\left.77.4\left(-\mathrm{C} \equiv \underline{\mathrm{C}}-\mathrm{CH}_{2}\right), 74.8\left(\mathrm{O}-\underline{\mathrm{C}}_{q}-\left(\mathrm{CH}_{2}\right)_{2} \mathrm{CH}_{3}\right), 61.2(\mathrm{C} \equiv \mathrm{C}-\underline{\mathrm{CH}})_{2}\right)$, $50.8\left(\mathrm{~N} 3-\mathrm{C}_{2}-\mathrm{CO}_{2}\right), 43.1\left(\mathrm{~N} 1-\mathrm{CH}_{2}-\mathrm{CO}_{2}\right), 40.1\left(\mathrm{CH}_{2}\right), 39.4$ $\left(\mathrm{CH}_{2}\right), 37.5\left(\mathrm{CH}_{2}\right), 37.4\left(\mathrm{CH}_{2}\right), 37.3\left(\mathrm{CH}_{2}\right), 32.8\left(\mathrm{CH} / \mathrm{CH}_{3}\right)$, $32.7\left(\mathrm{CH} / \mathrm{CH}_{3}\right), 31.2\left(\mathrm{C}_{\text {ar. }}-\mathrm{CH}_{2}-\mathrm{CH}_{2}\right), 28.0\left(\mathrm{O}-\mathrm{C}_{\mathrm{q}}-\mathrm{CH}_{3}\right), 27.9$ $\left(\mathrm{O}-\mathrm{C}_{\mathrm{q}}-\mathrm{CH}_{3}\right), 26.9 \quad\left(\mathrm{CH}_{2}\right), 24.8\left(\mathrm{CH}_{2}\right), 24.4 \quad\left(\mathrm{CH}_{2}\right), 23.8$ $\left(\mathrm{CH} / \mathrm{CH}_{3}\right), 22.8\left(\mathrm{CH} / \mathrm{CH}_{3}\right), 22.7\left(\mathrm{CH} / \mathrm{CH}_{3}\right), 21.0\left(\mathrm{CH}_{2}\right), 20.6$ $\left(\mathrm{CH}_{2}-\mathrm{CH}_{2}-\mathrm{C}_{\mathrm{q}}\right), \quad 19.8 \quad\left(\mathrm{CH} / \mathrm{CH}_{3}\right), \quad 19.7 \quad\left(\mathrm{CH} / \mathrm{CH}_{3}\right), \quad 19.6$ $\left(\mathrm{CH} / \mathrm{CH}_{3}\right), 13.1\left(\mathrm{C}_{\mathrm{ar}}-\underline{\mathrm{C}} \mathrm{H}_{3}\right), 12.3\left(\mathrm{C}_{\mathrm{ar}}-\underline{\mathrm{C}} \mathrm{H}_{3}\right), 11.8\left(\mathrm{C}_{\mathrm{ar}}-\underline{\mathrm{CH}_{3}}\right)$.

\section{(5-Iodouracil-1-yl) acetic acid (7) ${ }^{11}$}

$\mathrm{KOH}(3.62 \mathrm{~g}, 64.40 \mathrm{mmol})$ and 5-iodouracil 2 (4.00 $\mathrm{g}, 16.80 \mathrm{mmol})$ were dissolved in water $(40 \mathrm{~mL})$ and heated to $40{ }^{\circ} \mathrm{C}$. A solution of bromo acetic acid $((3.48 \mathrm{~g}, 25.20 \mathrm{mmol})$ in water $(10 \mathrm{~mL})$ was slowly added under stirring over a period of $30 \mathrm{~min}$. Stirring at $40{ }^{\circ} \mathrm{C}$ was continued for $30 \mathrm{~min}$. After cooling to room temperature the $\mathrm{pH}$ was adjusted to 5 by addition of conc. hydrochloric acid. After standing in a refrigerator for $2 \mathrm{~h}$ the precipitate of unreacted starting material 2 was filtered off. The filtrated was further acidified with conc. $\mathrm{HCl}$ to $\mathrm{pH} 2$. After $2 \mathrm{~h}$ the colorless product 7 was filtered off, washed with water and dried under vaccum. Yield $2.59 \mathrm{~g}(52 \%)$, colorless solid. ${ }^{1} \mathrm{H}-\mathrm{NMR}(300 \mathrm{MHz}$, DMSO$\left.\mathrm{D}_{6}\right): \delta(\mathrm{ppm})=11.74(\mathrm{~s}, 1 \mathrm{H}(\mathrm{NH} 3)), 8.20(\mathrm{~s}, 1 \mathrm{H}(\mathrm{CH} 6)), 4.40$ (s, $\left.2 \mathrm{H}\left(\mathrm{N} 1-\mathrm{CH}_{2}-\mathrm{CO}_{2}\right)\right) \cdot{ }^{13} \mathrm{C}-\mathrm{NMR}\left(75 \mathrm{MHz}, \mathrm{CDCl}_{3}\right): \delta(\mathrm{ppm})$ $=169.8\left(\mathrm{~N} 1-\mathrm{CH}_{2}-\mathrm{CO}_{2}\right), 161.5(\mathrm{O}=\mathrm{C} 4), 151.1(\mathrm{O}=\mathrm{C} 2), 150.7$ (CH6), $68.5\left(\mathrm{C}_{\mathrm{q}} \mathrm{-I}\right), 49.0\left(\mathrm{~N} 1-\underline{\mathrm{CH}}_{2}-\mathrm{CO}_{2}\right)$.

\section{2-(5-((Tocopher-6-yloxy)-prop-1-inyl)uracilyl) acetic acid (8) ${ }^{12}$}

$\mathrm{Pd}\left(\mathrm{PPh}_{3}\right)_{4}(0.07 \mathrm{~g}, 0.06 \mathrm{mmol})$ und $\mathrm{CuI}(0.04 \mathrm{~g}, 0.20$ mmol) were added to a solution of 5-iodouracil acetic acid 7 $(0.50 \mathrm{~g}, 1.69 \mathrm{mmol})$ in dry DMF $(5 \mathrm{~mL})$ under argon. A solution of (O-propargyl)-tocopherol $3(3.17 \mathrm{~g}, 6.76 \mathrm{mmol})$ in dry DMF $(6 \mathrm{~mL})$ and triethyl amine $(0.63 \mathrm{~mL}, 4.56 \mathrm{mmol})$ were added dropwise. After stirring at $\mathrm{rt}$ under argon for $2 \mathrm{~d}$ the solvent was stripped off under vacuum and the remainder purified by column chromatography $(300 \mathrm{~g}$ silica, cyclohexane/EtOAc/formic acid 1:1:0.5\%) affording $0.80 \mathrm{~g}$ (75 \%) product 8 as a sand-colored solid. $\mathrm{R}_{\mathrm{f}}=0.13$ (cyclohexane/EtOAc/formic acid 1:1:0.5\%). m. p. 145-148 ${ }^{\circ} \mathrm{C}$. Anal. calcd. for $\mathrm{C}_{36} \mathrm{H}_{56} \mathrm{~N}_{2} \mathrm{O}_{4}$ : C 71.67, $\mathrm{H}$ 8.86, $\mathrm{N}$ 4.40. Found $\mathrm{C} 72.25, \mathrm{H}$ 8.95, N 4.20. HRMS (ESI) $\mathrm{m} / \mathrm{z} \quad\left(\mathrm{M}+\mathrm{H}^{+}\right)$ $\mathrm{C}_{36} \mathrm{H}_{57} \mathrm{~N}_{2} \mathrm{O}_{4}$ calcd. 635.4060, found635.4050. ${ }^{1} \mathrm{H}-\mathrm{NMR} \quad(300$ $\left.\mathrm{MHz}, \mathrm{CDCl}_{3}\right): \delta(\mathrm{ppm})=11.76(\mathrm{~s}, 1 \mathrm{H}(\mathrm{NH} 3)), 8.12(\mathrm{~s}, 1 \mathrm{H}$

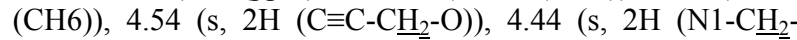
$\left.\left.\mathrm{CO}_{2}\right)\right), 2.49-2.52\left(\mathrm{~m}, 2 \mathrm{H}\left(\mathrm{O}-\mathrm{C}_{\mathrm{q}}-\mathrm{CH}_{2}-\mathrm{CH}_{2}-\mathrm{C}_{\mathrm{q}}\right)\right), 2.11(\mathrm{~s}, 3 \mathrm{H}$ $\left.\left(\mathrm{C}_{\mathrm{ar}}-\mathrm{CH}_{3}\right)\right), 2.09\left(\mathrm{~s}, 3 \mathrm{H}\left(\mathrm{C}_{\mathrm{ar}}-\mathrm{CH}_{3}\right)\right), 1.97\left(\mathrm{~s}, 3 \mathrm{H}\left(\mathrm{C}_{\mathrm{ar}}-\mathrm{CH}_{3}\right)\right)$, $1.63-1.77\left(\mathrm{~m}, 2 \mathrm{H}\left(\mathrm{O}-\mathrm{C}_{\mathrm{q}}-\mathrm{CH}_{2}-\mathrm{CH}_{2}-\mathrm{C}_{\mathrm{q}}\right)\right), 0.93-1.56(\mathrm{~m}, 24 \mathrm{H}$ $\left.\left(3 \times \mathrm{CH}, 9 \times \mathrm{CH}_{2}, 1 \times \mathrm{CH}_{3}\right)\right), 0.80-0.89\left(\mathrm{~m}, 12 \mathrm{H}\left(4 \times \mathrm{CH}-\mathrm{CH}_{3}\right)\right)$. ${ }^{13} \mathrm{C}-\mathrm{NMR}\left(75 \mathrm{MHz}, \mathrm{CDCl}_{3}\right): \delta(\mathrm{ppm})=169.6\left(-\mathrm{CO}_{2}\right), 162.4$
$(\mathrm{O}=\mathrm{C} 4), 150.4(\mathrm{O}=\mathrm{C} 2), 150.3(\mathrm{CH} 6), 147.9\left(\mathrm{O}-\underline{\mathrm{C}}_{\mathrm{ar}}\right), 127.7$ $\left.\left(\mathrm{C}_{\mathrm{ar}}\right), 126.1\left(\mathrm{C}_{\mathrm{ar} .}\right), 122.2\left(\mathrm{C}_{\mathrm{ar} .}\right), 117.7\left(\mathrm{C}_{\mathrm{ar} .}\right), 97.3\left(\mathrm{C}_{\mathrm{ar} .}\right), 8\right), 8$ $\left(-\underline{\mathrm{C}} \equiv \mathrm{C}-\mathrm{CH}_{2}\right), 78.8\left(-\mathrm{C} \equiv \underline{\mathrm{C}}-\mathrm{CH}_{2}\right), 74.8\left(\mathrm{O}-\underline{\mathrm{C}}_{\mathrm{q}}-\left(\mathrm{CH}_{2}\right)_{2} \mathrm{CH}_{3}\right), 61.2$ $\left(\mathrm{C} \equiv \mathrm{C}-\underline{\mathrm{CH}}_{2}\right), 49.2\left(\mathrm{~N} 1-\mathrm{CH}_{2}-\mathrm{CO}_{2}\right), 37.3\left(\mathrm{CH}_{2}\right), 37.2\left(\mathrm{CH}_{2}\right), 37.2$ $\left(\mathrm{CH}_{2}\right), 37.1\left(\mathrm{CH}_{2}\right), 32.5\left(\mathrm{CH} / \mathrm{CH}_{3}\right), 32.4\left(\mathrm{CH} / \mathrm{CH}_{3}\right), 31.2$ $\left(\mathrm{CH}_{2}\right), 31.2\left(\mathrm{C}_{\text {ar }}-\mathrm{CH}_{2}-\mathrm{CH}_{2}\right), 31.1\left(\mathrm{CH}_{2}\right), 27.8\left(\mathrm{CH} / \mathrm{CH}_{3}\right), 24.6$ $\left(\mathrm{CH}_{2}\right), 24.2\left(\mathrm{CH}_{2}\right), 23.8\left(\mathrm{CH} / \mathrm{CH}_{3}\right), 22.9\left(\mathrm{CH} / \mathrm{CH}_{3}\right), 22.8$ $\left(\mathrm{CH} / \mathrm{CH}_{3}\right), 20.8\left(\mathrm{CH}_{2}\right), 20.4\left(\mathrm{CH}_{2}-\mathrm{CH}_{2}-\mathrm{C}_{\mathrm{q}}\right), 19.9\left(\mathrm{CH} / \mathrm{CH}_{3}\right)$, $19.9\left(\mathrm{CH} / \mathrm{CH}_{3}\right), 13.3\left(\mathrm{C}_{\mathrm{ar}}-\underline{-} \mathrm{CH}_{3}\right), 12.4\left(\mathrm{C}_{\mathrm{ar}}-\underline{-} \mathrm{CH}_{3}\right), 12.0\left(\mathrm{C}_{\mathrm{ar}}-\right.$ $\left.\mathrm{CH}_{3}\right)$.

\section{1,3-Bis(4-Tocopherylmethyl-1,2,3-triazol-1-yl)-propan-2-ol} (11)

$\mathrm{NaN}_{3}(7.48 \mathrm{~g}, 115.00 \mathrm{mmol})$ was suspended in a $4: 1$ mixture of $\mathrm{DMF} /$ water $(40 \mathrm{ml})$ and combined with 1,3 dibromopropan-2-ol $(2.50 \mathrm{~g}, 11.50 \mathrm{mmol})$. The mixture was heated to $60{ }^{\circ} \mathrm{C}$ and stirred for 2 days. The resulting 1,3diazidopropan-2-ol $\mathbf{1 0}$ was not isolated but combined with a suspension of $O$-propargyltocopherol 3 (10.76 g, $23.00 \mathrm{mmol}$ ) in a 4: 1 mixture of DMF/water $(30 \mathrm{~mL})$ under argon. After the addition of $\mathrm{CuSO}_{4}$-monohydrate $(0.18 \mathrm{~g}, 1.15 \mathrm{mmol}), \mathrm{Na}$ ascorbate $(0.91 \mathrm{~g}, 4.60 \mathrm{mmol})$ und tris[(1-benzyl-1H-1,2,3triazol-4-yl)methyl]amine $(0.61 \mathrm{~g}, 1.15 \mathrm{mmol})$ under argon the mixture was stirred at $60{ }^{\circ} \mathrm{C}$ for $6 \mathrm{~d}$. The crude product 11 was dissolved in EtOAc and solid remainders were filtered off. The filtrate was concentrated under vacuum and the remainder was purified by column chromatography $(2000 \mathrm{~g}$ silica, cyclohexane/EtOAc 7:3) yielding $6.82 \mathrm{~g}(55 \%)$ of the product 11 as sticky yellow oil. $\mathrm{R}_{\mathrm{f}}=0.09$ (cyclohexane/EtOAc $7: 3$ ). HRMS (ESI) $m / z\left(\mathrm{M}+\mathrm{H}^{+}\right) \quad \mathrm{C}_{67} \mathrm{H}_{110} \mathrm{~N}_{6} \mathrm{O}_{5}$ calcd. 1079.8616, found 1079.8573. ${ }^{1} \mathrm{H}-\mathrm{NMR}\left(300 \mathrm{MHz}, \mathrm{CDCl}_{3}\right): \delta(\mathrm{ppm})=$ 7.84-8.02 (m, $2 \mathrm{H}(2 \times \mathrm{N}-\mathrm{CH})), 4.33-4.94(\mathrm{~m}, 9 \mathrm{H}(2 \times \mathrm{HC}=\mathrm{CN}-$ $\left.\mathrm{C}_{2}-\mathrm{O}, 2 \times \mathrm{N}-\underline{\mathrm{C}}_{2}-\mathrm{CH}, 1 \times \mathrm{CH}_{2}-\mathrm{CH}-\mathrm{CH}_{2}\right), 2.46-2.73(\mathrm{~m}, 4 \mathrm{H}$ $\left.\left(2 \times \mathrm{O}-\mathrm{C}_{\mathrm{q}}-\underline{\mathrm{C}}_{2}-\mathrm{CH}_{2}-\mathrm{C}_{\mathrm{q}}\right)\right), \quad 2.00-2.32\left(\mathrm{~m}, 18 \mathrm{H}\left(6 \times \mathrm{C}_{\mathrm{ar} .}-\mathrm{CH}_{3}\right)\right)$, $1.71-1.91\left(\mathrm{~m}, 4 \mathrm{H}\left(2 \times \mathrm{O}-\mathrm{C}_{\mathrm{q}}-\mathrm{CH}_{2}-\mathrm{CH}_{2}-\mathrm{C}_{\mathrm{q}}\right)\right), 1.02-1.69(\mathrm{~m}, 48 \mathrm{H}$ $\left.\left(6 \times \mathrm{CH}, 18 \times \mathrm{CH}_{2}, 2 \times \mathrm{CH}_{3}\right)\right), 0.79-1.04\left(\mathrm{~m}, 24 \mathrm{H}\left(8 \times \mathrm{CH}-\mathrm{CH}_{3}\right)\right)$. ${ }^{13} \mathrm{C}-\mathrm{NMR}\left(75 \mathrm{MHz}, \mathrm{CDCl}_{3}\right): \delta(\mathrm{ppm})=148.1\left(\mathrm{O}-\mathrm{C}_{\mathrm{ar}}\right), 14 \overline{7} .7$ $\left(\mathrm{CH}_{2}-\mathrm{CN}-\mathrm{CH}\right), 144.3\left(\mathrm{O}-\mathrm{C}_{\text {ar. }}\right), 127.7\left(\mathrm{C}_{\mathrm{ar}}\right), 125.8\left(\mathrm{C}_{\mathrm{ar} .}\right), 125.0$ $\left(\mathrm{CH}_{2}-\mathrm{CN}-\underline{\mathrm{CH}}-\mathrm{N}\right),\left(\mathrm{C} 22.9\left(\mathrm{C}_{\mathrm{ar}}\right), 117.5\left(\mathrm{C}_{\mathrm{ar}}\right), 74.7\left(\mathrm{O}-\underline{\mathrm{C}}_{\mathrm{q}^{-}}\right)\right.$ $\left.\left(\mathrm{CH}_{2}\right)_{2} \mathrm{CH}_{3}\right), 65.9(\mathrm{CH}-\mathrm{OH}), 60.3\left(\mathrm{NC}=\mathrm{CH}-\mathrm{CH}_{2}-\mathrm{O}\right), 53.2(\mathrm{~N}-$ $\left.\mathrm{CH}_{2}-\mathrm{CH}\right), 40.2\left(\mathrm{CH}_{2}\right), 39.4\left(\mathrm{CH}_{2}\right), 37.6\left(\mathrm{CH}_{2}\right), 37.5\left(\mathrm{CH}_{2}\right)$, $37.4\left(\mathrm{CH}_{2}\right), 37.4\left(\mathrm{CH}_{2}\right), 37.3\left(\mathrm{CH}_{2}\right), 32.7\left(\mathrm{CH} / \mathrm{CH}_{3}\right), 32.7$ $\left(\mathrm{CH} / \mathrm{CH}_{3}\right), 31.3\left(\mathrm{C}_{\mathrm{ar}}-\mathrm{CH}_{2}-\mathrm{CH}_{2}\right), 31.2\left(\mathrm{CH}_{2}\right), 27.9\left(\mathrm{CH} / \mathrm{CH}_{3}\right)$, $24.8\left(\mathrm{CH}_{2}\right), 24.4\left(\mathrm{CH}_{2}\right), 23.7\left(\mathrm{CH} / \mathrm{CH}_{3}\right), 22.7\left(\mathrm{CH} / \mathrm{CH}_{3}\right)$, $22.6\left(\mathrm{CH} / \mathrm{CH}_{3}\right), 21.1\left(\mathrm{CH}_{2}\right), 21.0\left(\mathrm{CH} / \mathrm{CH}_{3}\right), 20.9\left(\mathrm{CH}_{2}-\mathrm{CH}_{2}-\right.$ $\left.\mathrm{C}_{\mathrm{q}}\right), 20.6\left(\mathrm{CH}_{2}\right), 19.8\left(\mathrm{CH} / \mathrm{CH}_{3}\right), 19.7\left(\mathrm{CH} / \mathrm{CH}_{3}\right), 19.6$ $\left(\mathrm{CH} / \mathrm{CH}_{3}\right), 12.8\left(\mathrm{C}_{\mathrm{ar} .}-\underline{\mathrm{C}} \mathrm{H}_{3}\right), 12.0\left(\mathrm{C}_{\mathrm{ar}}-\underline{\mathrm{C}} \mathrm{H}_{3}\right), 11.8\left(\mathrm{C}_{\mathrm{ar}}-\underline{\mathrm{C}} \mathrm{H}_{3}\right)$.

\section{2-Propargyl-1,3-bis(4-tocopherylmethyl-1,2,3-triazol-1-yl)- propane (12)}

$\mathrm{NaH}(0.11 \mathrm{~g}, 4.65 \mathrm{mmol})$ was added to a solution of $\mathbf{1 1}$ $(4.56 \mathrm{~g}, 4.23 \mathrm{mmol})$ in dry THF $(150 \mathrm{~mL})$. After stirring at room temperature for $2 \mathrm{~h}$ propargyl bromide $(0.55 \mathrm{~g}$, $4.65 \mathrm{mmol}$ ) was added dropwise and stirring continued overnight. The solvent was removed under vacuum and the remainder dissolved in diethyl ether, washed with water (50 $\mathrm{mL})$ three times and with brine $(40 \mathrm{~mL})$ twice. After drying with $\mathrm{MgSO}_{4}$ the solvent was stripped off and the remainder purified by column chromatography $(500 \mathrm{~g}$ silica, cyclohexane/EtOAc $7: 3)$ yielding $4.17 \mathrm{~g}(88 \%)$ of the product 12 as yellow sticky oil. $\mathrm{R}_{\mathrm{f}}=0.25$ (cyclohexane / EtOAc $7: 3)$. HRMS (ESI) $m / z\left(\mathrm{M}+\mathrm{H}^{+}\right) \mathrm{C}_{70} \mathrm{H}_{112} \mathrm{~N}_{6} \mathrm{O}_{5}$ calcd. 1116.8694, found $1116.8663 .{ }^{1} \mathrm{H}-\mathrm{NMR}\left(300 \mathrm{MHz}, \mathrm{CDCl}_{3}\right): \delta$ $(\mathrm{ppm})=7.92(\mathrm{~s}, 2 \mathrm{H}(2 \times \mathrm{N}-\mathrm{CH})), 4.87\left(\mathrm{~s}, 4 \mathrm{H}\left(2 \times \mathrm{HC}=\mathrm{CN}-\underline{\mathrm{H}}_{2}-\right.\right.$ O)), 4.40-4.74 (m, 5H, $\left.2 \times \mathrm{N}-\mathrm{CH}_{2}-\mathrm{CH}, 1 \times \mathrm{CH}_{2}-\mathrm{C} \underline{\mathrm{H}}-\mathrm{CH}_{2}\right)$ ), 
4.03-4.20 (m, $\left.2 \mathrm{H}\left(\mathrm{HC} \equiv \mathrm{C}-\mathrm{CH}_{2}-\mathrm{O}\right)\right), 2.54-2.68(\mathrm{~m}, 4 \mathrm{H}(2 \times \mathrm{O}-$ $\left.\left.\mathrm{C}_{\mathrm{q}}-\mathrm{C}_{2}-\mathrm{CH}_{2}-\mathrm{C}_{\mathrm{q}}\right)\right), 2.51\left(\mathrm{~s}, 1 \mathrm{H}\left(\underline{\mathrm{HC}} \equiv \mathrm{C}-\mathrm{CH}_{2}-\mathrm{O}\right)\right), 2.24(\mathrm{~s}, 6 \mathrm{H}$ $\left.\left(2 \times \mathrm{C}_{\mathrm{ar} .}-\mathrm{CH}_{3}\right)\right), 2.20\left(\mathrm{~s}, 6 \mathrm{H}\left(2 \times \mathrm{C}_{\mathrm{ar}}-\mathrm{CH}_{3}\right)\right), 2.13\left(\mathrm{~s}, 6 \mathrm{H}\left(2 \times \mathrm{C}_{\mathrm{ar}}-\right.\right.$ $\left.\left.\mathrm{CH}_{3}\right)\right)$, 1.71-1.93 (m, 4H $\left.\left(2 \times \mathrm{O}-\mathrm{C}_{\mathrm{q}}-\mathrm{CH}_{2}-\mathrm{CH}_{2}-\mathrm{C}_{\mathrm{q}}\right)\right), 1.05-1.69$ $\left(\mathrm{m}, 48 \mathrm{H}\left(6 \times \mathrm{CH}, 18 \times \mathrm{CH}_{2}, 2 \times \mathrm{CH}_{3}\right)\right), 0.81-1.03(\mathrm{~m}, 24 \mathrm{H}$ $\left.\left(8 \times \mathrm{CH}-\mathrm{CH}_{3}\right)\right) \cdot{ }^{13} \mathrm{C}-\mathrm{NMR}\left(75 \mathrm{MHz}, \mathrm{CDCl}_{3}\right): \delta(\mathrm{ppm})=148.1$ $\left(\mathrm{O}-\mathrm{C}_{\text {ar }}\right), 147.8\left(\mathrm{CH}_{2}-\underline{\mathrm{CN}}-\mathrm{CH}\right), 144.8\left(\mathrm{O}-\mathrm{C}_{\text {ar }}\right), 127.8\left(\mathrm{C}_{\text {ar }}\right)$, $125.8\left(\mathrm{C}_{\mathrm{ar}}\right), 124.7\left(\mathrm{CH}_{2}-\mathrm{CN}-\underline{\mathrm{C}} \mathrm{H}-\mathrm{N}\right), 122.9\left(\mathrm{C}_{\mathrm{ar} .}\right), 117.5\left(\mathrm{C}_{\mathrm{ar} .}\right)$, $78.6\left(\mathrm{CH}-\mathrm{O}-\mathrm{CH}_{2}\right), 76.1\left(\mathrm{HC} \equiv \underline{\mathrm{C}}-\mathrm{CH}_{2}-\mathrm{O}\right), 76.0 \quad\left(\mathrm{HC} \equiv \mathrm{C}^{-\mathrm{CH}_{2}-}\right.$ $\mathrm{O}), \quad 74.8\left(\mathrm{O}-\underline{\mathrm{C}}_{\mathrm{q}}-\left(\mathrm{CH}_{2}\right)_{2} \mathrm{CH}_{3}\right), \quad 66.2\left(\mathrm{NC}=\mathrm{CH}-\mathrm{CH}_{2}-\mathrm{O}\right), \quad 58.1$ $\left(\mathrm{HC} \equiv \mathrm{C}-\mathrm{CH}_{2}-\mathrm{O}\right), 50.6\left(\mathrm{~N}-\mathrm{CH}_{2}-\mathrm{CH}\right), 40.1\left(\mathrm{CH}_{2}\right), 39.4\left(\mathrm{CH}_{2}\right)$, $37.6\left(\mathrm{CH}_{2}\right), 37.5\left(\mathrm{CH}_{2}\right), 37.4\left(\mathrm{CH}_{2}\right), 37.3\left(\mathrm{CH}_{2}\right), 37.3\left(\mathrm{CH}_{2}\right)$, $32.8\left(\mathrm{CH} / \mathrm{CH}_{3}\right), 32.7\left(\mathrm{CH} / \mathrm{CH}_{3}\right), 31.2\left(\mathrm{C}_{\mathrm{arr}}-\mathrm{CH}_{2}-\mathrm{CH}_{2}\right), 31.2$ $\left(\mathrm{CH}_{2}\right) 28.0\left(\mathrm{CH} / \mathrm{CH}_{3}\right), 26.9\left(\mathrm{CH}_{2}\right), 24.8\left(\mathrm{CH}_{2}\right), 24.4\left(\mathrm{CH}_{2}\right)$, $23.8\left(\mathrm{CH} / \mathrm{CH}_{3}\right), 22.7\left(\mathrm{CH} / \mathrm{CH}_{3}\right), 22.6\left(\mathrm{CH} / \mathrm{CH}_{3}\right), 21.0\left(\mathrm{CH}_{2}\right)$, $20.6\left(\mathrm{CH}_{2}-\mathrm{CH}_{2}-\mathrm{C}_{\mathrm{q}}\right), 19.8\left(\mathrm{CH} / \mathrm{CH}_{3}\right), 19.7\left(\mathrm{CH} / \mathrm{CH}_{3}\right), 19.6$ $\left(\mathrm{CH} / \mathrm{CH}_{3}\right), 12.9\left(\mathrm{C}_{\text {ar. }} \underline{-} \mathrm{H}_{3}\right), 12.1\left(\mathrm{C}_{\mathrm{ar}}-\underline{\mathrm{C}} \mathrm{H}_{3}\right), 11.8,\left(\mathrm{C}_{\mathrm{ar}}-\underline{\mathrm{C}} \mathrm{H}_{3}\right)$.

Conjugate (13) of 2-propargyl-1,3-bis(4-tocopherylmethyl1,2,3-triazol-1-yl)-propane (12) with 5-iodouracil-1-yl) acetic acid (7)

$12(0.21 \mathrm{~g}, 0.89 \mathrm{mmol}), \mathrm{Pd}\left(\mathrm{PPh}_{3}\right)_{4}(0.04 \mathrm{~g}, 0.03 \mathrm{mmol})$ and $\mathrm{CuI}(0.02 \mathrm{~g}, 0.10 \mathrm{mmol})$ were dissolved in dry DMF $(4 \mathrm{ml})$ and put under argon. A solution of $7(4.00 \mathrm{~g}, 3.58 \mathrm{mmol})$ in dry DMF ( $8 \mathrm{ml})$ was added under argon. After $24 \mathrm{~h}$ stirring at room temperature, a solution containing product $\mathbf{1 3}$ was formed. $\mathrm{R}_{\mathrm{f}}=0.23$ (cyclohexane / EtOAc 1 : 1). HRMS (ESI) $m / z\left(\mathrm{M}+\mathrm{H}^{+}\right) \mathrm{C}_{70} \mathrm{H}_{112} \mathrm{~N}_{6} \mathrm{O}_{5}$ calcd. 1313.9508, found 1313.9429 .

\section{O-(4- Bromobutyl)-tocopherol (14)}

The reaction was performed in a flask covered with aluminium foil in order to exclude light. $50 \%$ Aqueous $\mathrm{KOH}$ $(30 \mathrm{ml})$ and a small amount of tetrabutylammonium bromide were added to a solution of $\alpha$-tocopherol $(30.00 \mathrm{~g}, 69.65$ $\mathrm{mmol})$ in THF $(180 \mathrm{ml})$ at $0{ }^{\circ} \mathrm{C}$ under argon. 1,4Dibromobutane $(24.79 \mathrm{~mL}, \quad 208.96 \mathrm{mmol})$ was added dropwise at $0{ }^{\circ} \mathrm{C}$ and stirring. The solution was stirred at $0{ }^{\circ} \mathrm{C}$ for $30 \mathrm{~min}$ and at $\mathrm{rt}$ overnight. THF was distilled off under vacuum and the crude product was dissolved in EtOAc. The solution was washed with water $(75 \mathrm{~mL})$ and aqueous ammonium chloride. The aqueous phase was twice reextracted with EtOAc $(25 \mathrm{~mL})$ and the combined organic phases were dried over $\mathrm{MgSO}_{4}$. The solvent and unreacted, excess dibromo butane were removed at $65^{\circ} \mathrm{C}$ under reduced pressure providing product $\mathbf{1 4}$ as yellow oil in quantitative yield. $\mathrm{R}_{\mathrm{f}}=0.13$ (cyclohexane / AcOEt $19: 1$ ). HRMS (ESI) $m / z\left(\mathrm{M}+\mathrm{H}^{+}\right) \mathrm{C}_{36} \mathrm{H}_{55} \mathrm{~N}_{2} \mathrm{O}_{4}$ calcd. 565.3615, found 565. 3618. ${ }^{1} \mathrm{H}-\mathrm{NMR}\left(300 \mathrm{MHz}, \mathrm{CDCl}_{3}\right): \delta(\mathrm{ppm})=3.69(\mathrm{t}, J=6.2 \mathrm{~Hz}$, $\left.2 \mathrm{H}\left(-\mathrm{CH}_{2}-\mathrm{CH}_{2}-\mathrm{O}\right)\right), 3.54\left(\mathrm{t}, J=6.7 \mathrm{~Hz}, 2 \mathrm{H}\left(\mathrm{Br}^{-} \mathrm{CH}_{2}-\mathrm{CH}_{2}-\right)\right.$, $2.59\left(\mathrm{t}, J=6.7 \mathrm{~Hz}, 2 \mathrm{H}\left(\mathrm{O}-\mathrm{C}_{\mathrm{q}}-\mathrm{CH}_{2}-\mathrm{CH}_{2}-\mathrm{C}_{\mathrm{q}}\right)\right), 2.04-2.23(\mathrm{~m}$, $\left.11 \mathrm{H} 1 \times\left(\mathrm{Br}-\mathrm{CH}_{2}-\mathrm{C}_{2}-\mathrm{CH}_{2}\right),\left(3 \times \mathrm{C}_{\mathrm{ar}}-\mathrm{CH}_{3}\right)\right), 1.90-2.04(\mathrm{~m}, 2 \mathrm{H}$, $\left(-\mathrm{CH}_{2}-\mathrm{CH}_{2}-\mathrm{O}\right), 1.71-1.89\left(\mathrm{~m}, 2 \mathrm{H}\left(\mathrm{O}-\mathrm{C}_{\mathrm{q}}-\mathrm{CH}_{2}-\mathrm{CH}_{2}-\mathrm{C}_{\mathrm{q}}\right)\right), 1.06-$ $1.65\left(\mathrm{~m}, 24 \mathrm{H}\left(3 \times \mathrm{CH}, 9 \times \mathrm{CH}_{2}, 1 \times \mathrm{C}-\mathrm{CH}_{3}\right)\right), 0.83-0.93(\mathrm{~m}, 12 \mathrm{H}$ $\left.\left(4 \times \mathrm{CH}^{-\mathrm{CH}_{3}}\right)\right) \cdot{ }^{13} \mathrm{C}-\mathrm{NMR}\left(75 \mathrm{MHz}, \mathrm{CDCl}_{3}\right): \delta(\mathrm{ppm})=148.2$ $\left(\mathrm{O}-\mathrm{C}_{\mathrm{ar}}\right), 147.8\left(\mathrm{O}-\mathrm{C}_{\mathrm{ar}}\right), 128.0\left(\mathrm{C}_{\mathrm{ar} .}\right), 126.1\left(\mathrm{C}_{\mathrm{ar}}\right), 123.0\left(\mathrm{C}_{\mathrm{ar} .}\right)$, 117.6 $\left(\mathrm{C}_{\mathrm{ar}}\right), 127.7\left(\mathrm{C}_{\mathrm{ar} .}\right), 125.7\left(\mathrm{C}_{\mathrm{ar} .}\right), 122.9\left(\mathrm{C}_{\mathrm{ar} .}\right), 117.5\left(\mathrm{C}_{\mathrm{ar} .}\right)$, $74.8\left(\mathrm{O}-\underline{\mathrm{C}}_{\mathrm{q}}-\left(\mathrm{CH}_{2}\right)_{2} \mathrm{CH}_{3}\right), 71.8\left(\mathrm{CH}_{2}-\mathrm{CH}_{2}-\mathrm{O}-\mathrm{C}_{\mathrm{ar}}\right), 40.1\left(\mathrm{CH}_{2}\right)$, $39.4\left(\mathrm{CH}_{2}\right), 37.6\left(\mathrm{CH}_{2}\right), 37.5\left(\mathrm{CH}_{2}\right), 37.4\left(\mathrm{CH}_{2}\right), 37.3\left(\mathrm{CH}_{2}\right)$, $33.7\left(\mathrm{Br}-\mathrm{CH}_{2}-\mathrm{CH}_{2}\right), 32.8\left(\mathrm{CH} / \mathrm{CH}_{3}\right), 32.7\left(\mathrm{CH} / \mathrm{CH}_{3}\right), 31.3(\mathrm{Br}-$ $\left.\mathrm{CH}_{2}-\underline{\mathrm{CH}}_{2}\right), 31.2\left(\mathrm{CH}_{2}\right), 29.8\left(\mathrm{C}_{\mathrm{arr}}-\underline{-} \mathrm{CH}_{2}-\mathrm{CH}_{2}\right), 29.0\left(\mathrm{CH}_{2}-\mathrm{CH}_{2}-\right.$ O-C $\left.\mathrm{C}_{\text {ar. }}\right), 28.0\left(\mathrm{CH} / \mathrm{CH}_{3}\right), 24.8\left(\mathrm{CH}_{2}\right), 24.4\left(\mathrm{CH}_{2}\right), 23.9$ $\left(\mathrm{CH} / \mathrm{CH}_{3}\right), 22.7\left(\mathrm{CH} / \mathrm{CH}_{3}\right), 22.6\left(\mathrm{CH} / \mathrm{CH}_{3}\right), 21.0\left(\mathrm{CH}_{2}\right), 20.6$ $\left(\mathrm{CH}_{2}-\mathrm{CH}_{2}-\mathrm{C}_{\mathrm{q}}\right), 19.7\left(\mathrm{CH} / \mathrm{CH}_{3}\right), 19.6\left(\mathrm{CH} / \mathrm{CH}_{3}\right), 12.7\left(\mathrm{C}_{\mathrm{ar} .}\right.$ $\left.\underline{\mathrm{CH}}_{3}\right), 11.9\left(\mathrm{C}_{\mathrm{ar} .}-\underline{\mathrm{C}} \mathrm{H}_{3}\right), 11.8\left(\mathrm{C}_{\mathrm{ar}}-\underline{\mathrm{C}} \mathrm{H}_{3}\right)$.

\section{O-(4-Phthalimidobutyl)-tocopherol (15)}

K-Phthalimide in dry DMF $(50 \mathrm{ml})$ was added to a solution of $O$-(4-brombutyl)-tocopherol $\mathbf{1 4}$ in dry DMF (100 $\mathrm{mL}$ ) under argon. The mixture was heated to $60 \mathrm{C}$ and stirred overnight, cooled to $\mathrm{rt}$ and concentrated to $30 \mathrm{~mL}$ under vacuum. Water $(120 \mathrm{~mL})$ was added and the product extracted twice with dichloromethane $(50 \mathrm{~mL})$. The organic layer was washed with $0.1 \mathrm{~N} \mathrm{NaOH}(80 \mathrm{ml})$ three times and brine. After drying with $\mathrm{MgSO}_{4}$ the solvent was removed under vacuum. Product 15 was obtained in quantitative yield as yellowish oil. HRMS (ESI) $m / z\left(\mathrm{M}+\mathrm{H}^{+}\right) \mathrm{C}_{41} \mathrm{H}_{62} \mathrm{NO}_{4}$ calcd.32.4673, found 632.4665. ${ }^{1} \mathrm{H}-\mathrm{NMR}\left(300 \mathrm{MHz}, \mathrm{CDCl}_{3}\right): \delta(\mathrm{ppm})=7.85\left(\mathrm{dd}, J_{1}\right.$ $\left.=5.5 \mathrm{~Hz}, J_{2}=3.0 \mathrm{~Hz}, 2 \mathrm{H}\left(\mathrm{CH}_{\text {ar }}\right)\right), 7.70\left(\mathrm{dd}, J_{1}=5.5 \mathrm{~Hz}, J_{2}=\right.$ $\left.3.0 \mathrm{~Hz}, 2 \mathrm{H}\left(\mathrm{CH}_{\mathrm{ar}}\right)\right), 3.80\left(\mathrm{t}, J=6.9 \mathrm{~Hz}, 2 \mathrm{H}\left(\mathrm{N}^{-} \underline{\mathrm{H}}_{2}-\mathrm{CH}_{2}-\right)\right)$, $3.66\left(\mathrm{t}, J=6.1 \mathrm{~Hz}, 2 \mathrm{H}\left(-\mathrm{CH}_{2}-\mathrm{CH}_{2}-\mathrm{O}\right)\right), 2.57$ (t, $J=6.6 \mathrm{~Hz}, 2 \mathrm{H}$ $\left.\left(\mathrm{O}-\mathrm{C}_{\mathrm{q}}-\mathrm{C}_{2}-\mathrm{CH}_{2}-\mathrm{C}_{\mathrm{q}}\right)\right), 2.16\left(\mathrm{~s}, 3 \mathrm{H}\left(\mathrm{C}_{\mathrm{ar}}-\mathrm{CH}_{3}\right)\right), 2.11(\mathrm{~s}, 3 \mathrm{H}$ $\left.\left(\mathrm{C}_{\mathrm{ar} .}-\mathrm{CH}_{3}\right)\right), 2.08\left(\mathrm{~s}, 3 \mathrm{H}\left(\mathrm{C}_{\mathrm{arr}}-\mathrm{CH}_{3}\right)\right), 1.89-2.00\left(\mathrm{~m}, 2 \mathrm{H}\left(\mathrm{N}^{-} \mathrm{CH}_{2}-\right.\right.$ $\left.\left.\mathrm{CH}_{2}-\mathrm{CH}_{2}\right)\right), 1.82-1.89\left(\mathrm{~m}, 2 \mathrm{H},\left(-\mathrm{CH}_{2}-\mathrm{CH}_{2}-\mathrm{O}\right), 1.70-1.82(\mathrm{~m}\right.$, $2 \mathrm{H}\left(\mathrm{O}-\mathrm{C}_{\mathrm{q}}-\mathrm{CH}_{2}-\mathrm{CH}_{2}-\mathrm{C}_{\mathrm{q}}\right), 0.98-1.62\left(\mathrm{~m}, 24 \mathrm{H}\left(3 \times \mathrm{CH}, 9 \times \mathrm{CH}_{2}\right.\right.$, $\left.\left.1 \times \mathrm{C}-\mathrm{CH}_{3}\right)\right), 0.80-0.90\left(\mathrm{~m}, 12 \mathrm{H}\left(4 \times \mathrm{CH}-\mathrm{CH}_{3}\right)\right) .{ }^{13} \mathrm{C}-\mathrm{NMR}(75$ $\left.\mathrm{MHz}, \mathrm{CDCl}_{3}\right): \delta(\mathrm{ppm})=168.4\left(\mathrm{~N}-\underline{\mathrm{CO}}-\mathrm{C}_{\mathrm{q}}^{-}\right), 148.1\left(\mathrm{O}-\underline{\mathrm{C}}_{\mathrm{ar}}\right)$, $147.7\left(\mathrm{O}-\underline{\mathrm{C}}_{\mathrm{ar}}\right), 133.9\left(\underline{\mathrm{CH}}_{\mathrm{ar}}\right), 132.2\left(\underline{\mathrm{C}}_{\mathrm{ar}}\right), 127.8\left(\mathrm{C}_{\mathrm{ar}}\right), 125.8$ $\left(\mathrm{C}_{\text {ar. }}\right), 123.2\left(\mathrm{CH}_{\mathrm{ar} .}\right), 122.8\left(\mathrm{C}_{\mathrm{ar} .}\right), 117.5\left(\mathrm{C}_{\mathrm{ar}}\right), 74.7\left(\mathrm{O}-\mathrm{C}_{\mathrm{q}}{ }^{-}\right.$ $\left.\left(\mathrm{CH}_{2}\right)_{2} \mathrm{CH}_{3}\right), 72.1\left(\mathrm{CH}_{2}-\mathrm{CH}_{2}-\mathrm{O}-\mathrm{C}_{\mathrm{ar}}\right), 40.1\left(\mathrm{CH}_{2}\right), 39.4\left(\mathrm{CH}_{2}\right)$, $38.0\left(\mathrm{~N}-\mathrm{CH}_{2}\right), 37.5\left(\mathrm{CH}_{2}\right), 37.4\left(\mathrm{CH}_{2}\right), 37.3\left(\mathrm{CH}_{2}\right), 32.8$ $\left(\mathrm{CH} / \mathrm{CH}_{3}\right), \quad 32.7\left(\mathrm{CH} / \mathrm{CH}_{3}\right), \quad 31.3\left(\mathrm{CH}_{2}-\mathrm{CH}_{2}-\mathrm{O}-\mathrm{C}_{\mathrm{ar}}\right), 28.0$ $\left(\mathrm{CH} / \mathrm{CH}_{3}\right), 27.8\left(\mathrm{C}_{\mathrm{ar} .}-\mathrm{CH}_{2}-\mathrm{CH}_{2}\right), 25.7\left(\mathrm{~N}-\mathrm{CH}_{2}-\mathrm{CH}_{2}\right), 24.8$ $\left(\mathrm{CH}_{2}\right), \quad 24.7 \quad\left(\mathrm{CH}_{2}\right), \quad 24.5 \quad\left(\mathrm{CH}_{2}\right), \quad 23.9 \quad\left(\mathrm{CH} / \mathrm{CH}_{3}\right), 22.8$ $\left(\mathrm{CH} / \mathrm{CH}_{3}\right), 22.7\left(\mathrm{CH} / \mathrm{CH}_{3}\right), 21.1\left(\mathrm{CH}_{2}\right), 20.7\left(\mathrm{CH}_{2}-\mathrm{CH}_{2}-\mathrm{C}_{\mathrm{q}}\right)$, $19.8\left(\mathrm{CH} / \mathrm{CH}_{3}\right), 19.7\left(\mathrm{CH} / \mathrm{CH}_{3}\right), 12.8\left(\mathrm{C}_{\mathrm{ar}}-\underline{-} \mathrm{CH}_{3}\right), 11.9\left(\mathrm{C}_{\mathrm{ar} .}-\right.$ $\left.\underline{\mathrm{CH}}_{3}\right), 11.8\left(\mathrm{C}_{\mathrm{ar}}-\underline{-} \mathrm{CH}_{3}\right)$.

\section{O-(4-Aminobutyl)-tocopherol (16)}

A mixture of the phthalimid 15, $55 \%$ hydrazine monohydrate $(4.38 \mathrm{ml}, 88.40 \mathrm{mmol})$ and ethanol $(50 \mathrm{~mL})$ was refluxed for $3 \mathrm{~h}$. After cooling to rt the solvent was removed under vacuum and the remainder dissolved in diethyl ether (40 $\mathrm{mL})$. The solution was washed three times with $0.1 \mathrm{~N} \mathrm{NaOH}$ $(15 \mathrm{~mL})$ and with brine. After drying over $\mathrm{Na}_{2} \mathrm{SO}_{4}$ and removing the solvent $8.74 \mathrm{~g}(98 \%)$ of the product 16 were obtained. HRMS (ESI) $\mathrm{m} / z\left(\mathrm{M}+\mathrm{H}^{+}\right) \mathrm{C}_{33} \mathrm{H}_{60} \mathrm{NO}_{2}$ calcd. 502.4624, found 502.4660. ${ }^{1} \mathrm{H}-\mathrm{NMR}\left(300 \mathrm{MHz}, \mathrm{CDCl}_{3}\right): \delta$ $(\mathrm{ppm})=8.35\left(\mathrm{~s}-\mathrm{br}, 2 \mathrm{H}\left(\mathrm{N}_{2}\right), 3.64\left(\mathrm{t}, J=5.6 \mathrm{~Hz}, 2 \mathrm{H}\left(-\mathrm{CH}_{2}-\right.\right.\right.$ $\left.\left.\mathrm{CH}_{2}-\mathrm{O}\right)\right), 3.13$ (s-br, $\left.2 \mathrm{H}\left(\mathrm{H}_{2} \mathrm{~N}-\mathrm{CH}_{2}-\mathrm{CH}_{2}\right)\right), 2.53(\mathrm{t}, J=6.6 \mathrm{~Hz}$, $\left.2 \mathrm{H}\left(\mathrm{O}-\mathrm{C}_{\mathrm{q}}-\mathrm{CH}_{2}-\mathrm{CH}_{2}-\mathrm{C}_{\mathrm{q}}\right)\right), 1.96-2.20\left(\mathrm{~m}, 11 \mathrm{H}\left(1 \times \mathrm{H}_{2} \mathrm{~N}_{-}-\mathrm{CH}_{2}-\right.\right.$ $\left.\left.\mathrm{C}_{2}-\mathrm{CH}_{2}\right),\left(3 \times \mathrm{C}_{\text {ar. }}-\mathrm{CH}_{3}\right)\right), 1.82-1.96\left(\mathrm{~m}, 2 \mathrm{H},\left(-\mathrm{CH}_{2}-\mathrm{CH}_{2}-\mathrm{O}\right)\right.$, $1.64-1.83\left(\mathrm{~m}, 2 \mathrm{H}\left(\mathrm{O}-\mathrm{C}_{\mathrm{q}}-\mathrm{CH}_{2}-\mathrm{CH}_{2}-\mathrm{C}_{\mathrm{q}}\right)\right), 1.01-1.55(\mathrm{~m}, 24 \mathrm{H}$ $\left.\left(3 \times \mathrm{CH}, 9 \times \mathrm{CH}_{2}, 1 \times \mathrm{C}-\mathrm{CH}_{3}\right)\right), 0.81-0.89\left(\mathrm{~m}, 12 \mathrm{H}\left(4 \times \mathrm{CH}-\mathrm{CH}_{3}\right)\right)$. ${ }^{13} \mathrm{C}-\mathrm{NMR}\left(75 \mathrm{MHz}, \mathrm{CDCl}_{3}\right): \delta(\mathrm{ppm})=148.1\left(\mathrm{O}-\mathrm{C}_{\mathrm{ar}}\right), 127.6$ $\left(\mathrm{C}_{\mathrm{ar}}\right), 125.6\left(\mathrm{C}_{\mathrm{ar}}\right), 122.9\left(\mathrm{C}_{\mathrm{ar}}\right), 117.5\left(\mathrm{C}_{\mathrm{ar}}\right), 74.8\left(\mathrm{O}-\underline{\mathrm{C}}_{\mathrm{q}}{ }^{-}\right.$ $\left.\left(\mathrm{CH}_{2}\right)_{2} \mathrm{CH}_{3}\right), \quad 71.8\left(\mathrm{CH}_{2}-\mathrm{CH}_{2}-\mathrm{O}-\mathrm{C}_{\text {ar }}\right), 40.2\left(\mathrm{~N}^{-\mathrm{CH}_{2}}\right), 40.1$ $\left(\mathrm{CH}_{2}\right), 39.4\left(\mathrm{CH}_{2}\right), 37.5\left(\mathrm{CH}_{2}\right), 37.4\left(\mathrm{CH}_{2}\right), 37.3\left(\mathrm{CH}_{2}\right), 32.8$ $\left(\mathrm{CH} / \mathrm{CH}_{3}\right), \quad 32.7\left(\mathrm{CH} / \mathrm{CH}_{3}\right), 31.3\left(\mathrm{CH}_{2}-\mathrm{CH}_{2}-\mathrm{O}-\mathrm{C}_{\mathrm{ar}}\right), 31.2$ $\left(\mathrm{CH}_{2}\right), 28.0\left(\mathrm{CH} / \mathrm{CH}_{3}\right), 27.4\left(\mathrm{C}_{\mathrm{ar}}-\mathrm{CH}_{2}-\mathrm{CH}_{2}\right), 24.8\left(\mathrm{CH}_{2}\right), 24.8$ $\left(\mathrm{CH}_{2}\right), 24.5\left(\mathrm{CH}_{2}\right), 23.7\left(\mathrm{CH} / \mathrm{CH}_{3}\right), 22.7\left(\mathrm{CH} / \mathrm{CH}_{3}\right), 22.6$ $\left(\mathrm{CH} / \mathrm{CH}_{3}\right), 21.1\left(\mathrm{CH}_{2}\right), 20.6\left(\mathrm{CH}_{2}-\mathrm{CH}_{2}-\mathrm{C}_{\mathrm{q}}\right), 19.8\left(\mathrm{CH} / \mathrm{CH}_{3}\right)$, $19.7\left(\mathrm{CH} / \mathrm{CH}_{3}\right), 12.9\left(\mathrm{C}_{\mathrm{ar}}-{ }^{-} \mathrm{CH}_{3}\right), 12.0\left(\mathrm{C}_{\mathrm{ar}}-\underline{\mathrm{CH}_{3}}\right), 11.8\left(\mathrm{C}_{\mathrm{ar}}\right.$ $\left.\mathrm{CH}_{3}\right)$.

\section{4,6-Difluoro-2-(prop-2-yn-1-yl-amino)-1,3,5-triazine (18)}

Propargylamine $(0.64 \mathrm{~mL}, 10.00 \mathrm{mmol})$ was added dropwise under argon to a solution of cyanuric fluoride $\mathbf{1 7}$ $(8.88 \mathrm{~mL}, 10.00 \mathrm{mmol})$ in dry DMF. The mixture was stirred overnight and the product isolated by column chromatography 
(250 g silica, cyclohexane / EtOAc $7: 3)$ yielding $1.66 \mathrm{~g} \mathrm{(98}$ $\%$ ) of 18 as crystalline solid. $\mathrm{R}_{\mathrm{f}}=0.43$ (cyclohexane / EtOAc $7: 3)$. HRMS (ESI) $m / z\left(\mathrm{M}+\mathrm{H}^{+}\right) \mathrm{C}_{6} \mathrm{H}_{4} \mathrm{~F}_{2} \mathrm{~N}_{4}$ calcd. 171.0482, found 171.0519. ${ }^{1} \mathrm{H}-\mathrm{NMR}\left(300 \mathrm{MHz}, \mathrm{CDCl}_{3}\right): \delta(\mathrm{ppm})=6.82$ $(\mathrm{s}, 1 \mathrm{H}(\mathrm{NH})), 4.30\left(\mathrm{dd}, J_{1}=5.7 \mathrm{~Hz}, J_{2}=2.6 \mathrm{~Hz}, 2 \mathrm{H}\left(\mathrm{CH}_{2}\right)\right)$, $2.32(\mathrm{t}, J=2.6,1 \mathrm{H}(\mathrm{CH})) .{ }^{13} \mathrm{C}-\mathrm{NMR}\left(75 \mathrm{MHz}, \mathrm{CDCl}_{3}\right): \delta$ $(\mathrm{ppm})=172.8\left(\mathrm{dd}, J_{1}=43.1 \mathrm{~Hz}, J_{2}=20.0 \mathrm{~Hz}, 1 \mathrm{C}(\mathrm{C}-\mathrm{F})\right)$, $169.8\left(\mathrm{dd}, J_{1}=42.6 \mathrm{~Hz}, J_{2}=20.0 \mathrm{~Hz}, 1 \mathrm{C}(\mathrm{C}-\mathrm{F})\right), 169.4\left(\mathrm{C}_{\mathrm{ar}}\right)$, $72.9\left(-\mathrm{CH}_{2}-\right), 31.4(-\mathrm{CH}) .{ }^{19} \mathrm{~F}-\mathrm{NMR}\left(282 \mathrm{MHz}, \mathrm{CDCl}_{3}\right): \delta$ $(\mathrm{ppm})=-35.80(\mathrm{~d}, J=13.9 \mathrm{~Hz}, 1 \mathrm{~F}),-38.37(\mathrm{~d}, J=13.9 \mathrm{~Hz}$, $1 \mathrm{~F})$.

\section{2-Propargylamino-4,6-bis(tocopherylbutylamino)-1,3,5-} triazine (19)

A solution of $16(3.00 \mathrm{~g}, 10.00 \mathrm{mmol})$ in toluene $(10 \mathrm{~mL})$ was added dropwise to a solution of $\mathbf{1 8}(0.34 \mathrm{~g}, 5.98 \mathrm{mmol})$ in toluene $(5 \mathrm{~mL})$. After refluxing for $4 \mathrm{~h}$ the mixture was stirred for 3 d. After chromatographic separation (300 silica, dichloromethane / methanol 95 : 5) $2.08 \mathrm{~g}$ (92\%) of the product 19 were obtained as yellow sticky oil. $\mathrm{R}_{\mathrm{f}}=0.69$
(DCM / MeOH / formic acid 89.50: $10: 0.5 \%$ ). HRMS (ESI) $m / z\left(\mathrm{M}+\mathrm{H}^{+}\right) \mathrm{C}_{72} \mathrm{H}_{121} \mathrm{~N}_{6} \mathrm{O}_{4}$ calcd. 1133.9449, found 1133.9429 . ${ }^{1} \mathrm{H}-\mathrm{NMR}\left(300 \mathrm{MHz}, \mathrm{CDCl}_{3}\right): \delta(\mathrm{ppm})=4.23$ (s-br, $2 \mathrm{H}$ $\left.\left(\mathrm{HC} \equiv \mathrm{C}-\mathrm{CH}_{2}-\mathrm{NH}\right)\right), 3.70$ (s-br, $\left.4 \mathrm{H}\left(2 \times-\mathrm{CH}_{2}-\mathrm{CH}_{2}-\mathrm{O}\right)\right), 3.52$ (sbr, $\left.4 \mathrm{H}\left(2 \times \overline{\mathrm{HN}}-\underline{\mathrm{C}}_{2}-\mathrm{CH}_{2}\right)\right), 2.54-2.67\left(\mathrm{~m}, 4 \mathrm{H}\left(2 \times \mathrm{O}-\mathrm{C}_{\mathrm{q}}-\mathrm{CH}_{2}-\right.\right.$ $\left.\left.\mathrm{CH}_{2}-\mathrm{C}_{\mathrm{q}}\right)\right), \quad 2.08-2.26\left(\mathrm{~m}, 19 \mathrm{H}\left(1 \times \underline{\mathrm{HC}} \equiv \mathrm{C}-\mathrm{CH}_{2}-\mathrm{NH}, \quad 6 \times \mathrm{C}_{\mathrm{ar}}-\right.\right.$ $\left.\left.\mathrm{CH}_{3}\right)\right), 1.72-2.00\left(\mathrm{~m}, 12 \mathrm{H}\left(2 \times \mathrm{HN}-\mathrm{CH}_{2}-\mathrm{C}_{2}-\mathrm{CH}_{2}\right),\left(2 \times \mathrm{CH}_{2}-\right.\right.$ $\left.\left.\mathrm{CH}_{2}-\mathrm{O}\right),\left(2 \times \mathrm{O}-\mathrm{C}_{\mathrm{q}}-\mathrm{CH}_{2}-\mathrm{CH}_{2}-\mathrm{C}_{\mathrm{q}}\right)\right), 1.06-1.67(\mathrm{~m}, 48 \mathrm{H}(6 \times \mathrm{CH}$, $\left.\left.18 \times \mathrm{CH}_{2}, 2 \times \mathrm{CH}_{3}\right)\right), \quad 0.86-0.97\left(\mathrm{~m}, 24 \mathrm{H}\left(8 \times \mathrm{CH}_{-} \mathrm{CH}_{3}\right)\right) .{ }^{13} \mathrm{C}-$ NMR $\left(75 \mathrm{MHz}, \mathrm{CDCl}_{3}\right): \delta(\mathrm{ppm})=166.0\left(\mathrm{HN}-\mathrm{C}_{\mathrm{ar}}^{-}\right), 165.6$ $\left(\mathrm{HN}-\mathrm{C}_{\text {ar }}\right), 148.3\left(\mathrm{O}-\mathrm{C}_{\mathrm{ar}}\right), 147.7\left(\mathrm{O}-\mathrm{C}_{\mathrm{ar}}\right), 127.8\left(\mathrm{C}_{\mathrm{ar} .}\right), 125.7$ $\left(\mathrm{C}_{\mathrm{ar}}\right), 122.8\left(\mathrm{C}_{\mathrm{ar}}\right), 117.4 \quad\left(\mathrm{C}_{\mathrm{ar}}\right), 74.7\left(\mathrm{O}_{-} \underline{\mathrm{C}}_{\mathrm{q}}-\left(\mathrm{CH}_{2}\right)_{2} \mathrm{CH}_{3}\right)$, $72.5(\underline{\mathrm{C}} \equiv \mathrm{CH}), 70.8\left(\mathrm{CH}_{2}-\mathrm{CH}_{2}-\mathrm{O}-\mathrm{C}_{\mathrm{ar}}\right), 40.6\left(\mathrm{CH}_{2}\right), 40.1\left(\mathrm{CH}_{2}\right)$, $39.4\left(\mathrm{CH}_{2}\right), 37.6\left(\mathrm{CH}_{2}\right), 37.5\left(\mathrm{CH}_{2}\right), 37.4\left(\mathrm{CH}_{2}\right), 37.3\left(\mathrm{CH}_{2}\right)$, $32.8\left(\mathrm{CH} / \mathrm{CH}_{3}\right), 32.7\left(\mathrm{CH} / \mathrm{CH}_{3}\right), 31.3\left(\mathrm{CH}_{2}\right), 31.3\left(\mathrm{C}_{\mathrm{ar}}-\mathrm{CH}_{2}-\right.$ $\left.\mathrm{CH}_{2}\right), 30.3\left(\mathrm{CH}_{2}\right), 28.0\left(\mathrm{CH} / \mathrm{CH}_{3}\right), 27.8\left(\mathrm{CH}_{2}\right), 26.9\left(\mathrm{CH}_{2}\right)$, $24.9\left(\mathrm{CH}_{2}\right), 24.5\left(\mathrm{CH}_{2}\right), 23.9\left(\mathrm{CH}_{2}\right), 22.8\left(\mathrm{CH} / \mathrm{CH}_{3}\right), 22.7$ $\left(\mathrm{CH} / \mathrm{CH}_{3}\right), 21.1\left(\mathrm{CH}_{2}\right), 20.7\left(\mathrm{CH}_{2}-\mathrm{CH}_{2}-\mathrm{C}_{\mathrm{q}}\right), 19.8\left(\mathrm{CH} / \mathrm{CH}_{3}\right)$, $19.7\left(\mathrm{CH} / \mathrm{CH}_{3}\right), 19.6\left(\mathrm{CH} / \mathrm{CH}_{3}\right), 12.8\left(\mathrm{C}_{\mathrm{ar} .}-\mathrm{CH}_{3}\right), 12.0\left(\mathrm{C}_{\mathrm{ar} .}\right.$ $\left.\underline{\mathrm{CH}}_{3}\right), 11.9\left(\mathrm{C}_{\mathrm{ar}}-\underline{\mathrm{C}} \mathrm{H}_{3}\right)$.

\section{X-Ray crystal analysis of 2-propargylamino-4,6-difluorotriazine (18)}

Table 1

Crystal data and structure refinement

Empirical formula

Formula weight

Temperature

Wavelength

Crystal system, space group

Unit cell dimensions

Volume

$Z$, Calculated density

Absorption coefficient

$\mathrm{F}(000)$

Crystal size

Theta range for data collection

Limiting indices

Reflections collected / unique

Completeness to theta $=27.49$

Absorption correction

Max. and min. transmission

Refinement method

Data / restraints / parameters

Goodness-of-fit on $\mathrm{F}^{\wedge} 2$

Final $\mathrm{R}$ indices [I $>2 \operatorname{sigma}(\mathrm{I})]$

$\mathrm{R}$ indices (all data)

Extinction coefficient

Largest diff. peak and hole

\author{
$\mathrm{C}_{6} \mathrm{H}_{4} \mathrm{~F}_{2} \mathrm{~N}_{4}$ \\ 170.13 \\ $100(2) \mathrm{K}$ \\ $0.71073 \AA$
}

Monoclinic, P 21/c

$\mathrm{a}=10.6922(15) \AA \mathrm{alpha}=90 \mathrm{deg}$.

$\mathrm{b}=4.9262(5) \AA$ beta $=93.263(11) \mathrm{deg}$.

$\mathrm{c}=13.3325(18) \AA$ gamma $=90 \mathrm{deg}$.

701.11(15) $\AA^{3}$

4, $1.612 \mathrm{Mg} / \mathrm{m}^{3}$

$0.144 \mathrm{~mm}^{-1}$

344

$0.50 \times 0.50 \times 0.20 \mathrm{~mm}$

3.06 to $27.49 \mathrm{deg}$.

$-13<=\mathrm{h}<=13,-6<=\mathrm{k}<=6,0<=1<=17$

$2904 / 1608$ [R(int $)=0.0205]$

$99.8 \%$

None

0.9717 and 0.9314

Full-matrix least-squares on $\mathrm{F}^{2}$

$1608 / 0 / 126$

1.288

$\mathrm{R} 1=0.0456, \mathrm{wR} 2=0.1236$

$\mathrm{R} 1=0.0531, \mathrm{wR} 2=0.1257$

$0.022(3)$

0.239 and -0.271 e. $\mathrm{A}^{-3}$ 


\section{Table 2}

Atomic coordinates $\left(\times 10^{4}\right)$ and equivalent isotropic displacement parameters $\left(\mathrm{A}^{2} \times 10^{3}\right)$. $\mathrm{U}(\mathrm{eq})$ is defined as one third of the trace of the orthogonalized Uij tensor

\begin{tabular}{lllll} 
& $\mathrm{x}$ & $\mathrm{y}$ & $\mathrm{z}$ & $\mathrm{U}(\mathrm{eq})$ \\
\hline $\mathrm{C}(1)$ & $8018(2)$ & $10461(5)$ & $6714(2)$ & $19(1)$ \\
$\mathrm{C}(2)$ & $9944(2)$ & $9679(5)$ & $6406(2)$ & $18(1)$ \\
$\mathrm{C}(3)$ & $8443(2)$ & $7144(5)$ & $5668(2)$ & $17(1)$ \\
$\mathrm{C}(4)$ & $4526(6)$ & $4769(2)$ & $22(1)$ \\
$\mathrm{C}(5)$ & $6787(2)$ & $6472(6)$ & $4044(2)$ & $21(1)$ \\
$\mathrm{C}(6)$ & $6216(2)$ & $8027(6)$ & $3461(2)$ & $29(1)$ \\
$\mathrm{F}(1)$ & $11934(3)$ & $7173(1)$ & $27(1)$ \\
$\mathrm{F}(2)$ & $10349(3)$ & $6517(1)$ & $22(1)$ \\
$\mathrm{N}(2)$ & $7147(3)$ & $11207(5)$ & $6909(2)$ & $20(1)$ \\
$\mathrm{N}(3)$ & $11151(1)$ & $7667(4)$ & $5794(1)$ & $17(1)$ \\
$\mathrm{N}(1)$ & $9192(2)$ & $8551(5)$ & $6123(2)$ & $19(1)$ \\
$\mathrm{N}(4)$ & $9695(2)$ & $5141(5)$ & $5046(2)$ & $19(1)$ \\
\hline
\end{tabular}

Table 3

Bond lengths $[\AA]$

Bond $\quad$ Length $[\AA]$

\begin{tabular}{|c|c|}
\hline $\mathrm{C}(1)-\mathrm{N}(1)$ & $1.309(3)$ \\
\hline $\mathrm{C}(1)-\mathrm{N}(2)$ & $1.319(3)$ \\
\hline $\mathrm{C}(1)-\mathrm{F}(1)$ & $1.328(3)$ \\
\hline $\mathrm{C}(2)-\mathrm{N}(3)$ & $1.301(3)$ \\
\hline $\mathrm{C}(2)-\mathrm{N}(2)$ & $1.313(3)$ \\
\hline$C(2)-F(2)$ & $1.332(3)$ \\
\hline $\mathrm{C}(3)-\mathrm{N}(4)$ & $1.327(3)$ \\
\hline $\mathrm{C}(3)-\mathrm{N}(1)$ & $1.352(3)$ \\
\hline $\mathrm{C}(3)-\mathrm{N}(3)$ & $1.364(3)$ \\
\hline $\mathrm{C}(4)-\mathrm{N}(4)$ & $1.464(3)$ \\
\hline$C(4)-C(5)$ & $1.469(4)$ \\
\hline $\mathrm{C}(4)-\mathrm{H}(2 \mathrm{a})$ & $0.96(3)$ \\
\hline $\mathrm{C}(4)-\mathrm{H}(2 \mathrm{~b})$ & $0.96(3)$ \\
\hline$C(5)-C(6)$ & $1.182(4)$ \\
\hline $\mathrm{C}(6)-\mathrm{H}(3)$ & $0.88(4)$ \\
\hline $\mathrm{N}(4)-\mathrm{H}(1)$ & $0.93(4)$ \\
\hline
\end{tabular}




\section{Table 4}

Bond angles [deg]

\begin{tabular}{|c|c|}
\hline Bond & Angle \\
\hline $\mathrm{N}(1)-\mathrm{C}(1)-\mathrm{N}(2)$ & $130.3(2)$ \\
\hline $\mathrm{N}(1)-\mathrm{C}(1)-\mathrm{F}(1)$ & 115.2(2) \\
\hline $\mathrm{N}(2)-\mathrm{C}(1)-\mathrm{F}(1)$ & $114.5(2)$ \\
\hline $\mathrm{N}(3)-\mathrm{C}(2)-\mathrm{N}(2)$ & $130.3(2)$ \\
\hline $\mathrm{N}(3)-\mathrm{C}(2)-\mathrm{F}(2)$ & $114.9(2)$ \\
\hline $\mathrm{N}(2)-\mathrm{C}(2)-\mathrm{F}(2)$ & $114.7(2)$ \\
\hline $\mathrm{N}(4)-\mathrm{C}(3)-\mathrm{N}(1)$ & 119.0(2) \\
\hline $\mathrm{N}(4)-\mathrm{C}(3)-\mathrm{N}(3)$ & $116.8(2)$ \\
\hline $\mathrm{N}(1)-\mathrm{C}(3)-\mathrm{N}(3)$ & $124.2(2)$ \\
\hline $\mathrm{N}(4)-\mathrm{C}(4)-\mathrm{C}(5)$ & $112.8(2)$ \\
\hline $\mathrm{N}(4)-\mathrm{C}(4)-\mathrm{H}(2 \mathrm{a})$ & $108.0(18)$ \\
\hline $\mathrm{C}(5)-\mathrm{C}(4)-\mathrm{H}(2 \mathrm{a})$ & $108.5(19)$ \\
\hline $\mathrm{N}(4)-\mathrm{C}(4)-\mathrm{H}(2 \mathrm{~b})$ & 107.4(18) \\
\hline $\mathrm{C}(5)-\mathrm{C}(4)-\mathrm{H}(2 \mathrm{~b})$ & $112.4(18)$ \\
\hline $\mathrm{H}(4 \mathrm{~A})-\mathrm{C}(4)-\mathrm{H}(1 \mathrm{~b})$ & 107(3) \\
\hline$C(6)-C(5)-C(4)$ & $179.5(3)$ \\
\hline $\mathrm{C}(5)-\mathrm{C}(6)-\mathrm{H}(3)$ & $178(2)$ \\
\hline $\mathrm{C}(2)-\mathrm{N}(2)-\mathrm{C}(1)$ & $110.0(2)$ \\
\hline$C(2)-N(3)-C(3)$ & $112.7(2)$ \\
\hline $\mathrm{C}(1)-\mathrm{N}(1)-\mathrm{C}(3)$ & $112.4(2)$ \\
\hline $\mathrm{C}(3)-\mathrm{N}(4)-\mathrm{C}(4)$ & $122.5(2)$ \\
\hline $\mathrm{C}(3)-\mathrm{N}(4)-\mathrm{H}(1)$ & $117(2)$ \\
\hline $\mathrm{C}(4)-\mathrm{N}(4)-\mathrm{H}(1)$ & $120(2)$ \\
\hline
\end{tabular}

Symmetry transformations used to generate equivalent atoms: 
Table 5

Anisotropic displacement parameters $\left(\mathrm{A}^{2} \times 10^{3}\right)$. The anisotropic displacement factor exponent takes the form: $-2 \pi^{2}\left[\mathrm{~h}^{2} \mathrm{a}^{2} \mathrm{U} 11+\ldots\right.$ $+2 \mathrm{~h} \mathrm{k} \mathrm{a}^{*} \mathrm{~b} * \mathrm{U} 12$ ]

\begin{tabular}{lllllll} 
& $\mathrm{U} 11$ & $\mathrm{U} 22$ & $\mathrm{U} 33$ & $\mathrm{U} 23$ & $\mathrm{U} 13$ & $\mathrm{U} 12$ \\
\hline $\mathrm{C}(1)$ & $19(1)$ & $21(1)$ & $19(1)$ & $3(1)$ & $3(1)$ & $3(1)$ \\
$\mathrm{C}(2)$ & $16(1)$ & $21(1)$ & $17(1)$ & $6(1)$ & $-2(1)$ & $-2(1)$ \\
$\mathrm{C}(3)$ & $15(1)$ & $19(1)$ & $16(1)$ & $5(1)$ & $0(1)$ & $-1(1)$ \\
$\mathrm{C}(4)$ & $17(1)$ & $23(1)$ & $24(1)$ & $0(1)$ & $-1(1)$ & $-4(1)$ \\
$\mathrm{C}(5)$ & $15(1)$ & $23(1)$ & $26(1)$ & $-4(1)$ & $-1(1)$ & $-4(1)$ \\
$\mathrm{C}(6)$ & $24(1)$ & $28(2)$ & $34(2)$ & $3(1)$ & $-8(1)$ & $-3(1)$ \\
$\mathrm{F}(1)$ & $21(1)$ & $30(1)$ & $30(1)$ & $-8(1)$ & $4(1)$ & $4(1)$ \\
$\mathrm{F}(2)$ & $16(1)$ & $26(1)$ & $25(1)$ & $2(1)$ & $-1(1)$ & $-5(1)$ \\
$\mathrm{N}(2)$ & $20(1)$ & $19(1)$ & $21(1)$ & $0(1)$ & $-2(1)$ & $-1(1)$ \\
$\mathrm{N}(3)$ & $15(1)$ & $20(1)$ & $17(1)$ & $3(1)$ & $0(1)$ & $0(1)$ \\
$\mathrm{N}(1)$ & $16(1)$ & $21(1)$ & $20(1)$ & $1(1)$ & $1(1)$ & $0(1)$ \\
$\mathrm{N}(4)$ & $15(1)$ & $21(1)$ & $20(1)$ & $-2(1)$ & $0(1)$ & $0(1)$ \\
\hline
\end{tabular}

Table 6

Hydrogen coordinates $\left(\times 10^{4}\right)$ and isotropic displacement parameters $\left(\mathrm{A}^{2} \times 10^{3}\right)$

\begin{tabular}{lllll} 
& $\mathrm{x}$ & $\mathrm{y}$ & $\mathrm{z}$ & $\mathrm{U}(\mathrm{eq})$ \\
\hline $\mathrm{H}(2 \mathrm{a})$ & $6330(30)$ & $4580(70)$ & $5370(20)$ & $26(8)$ \\
$\mathrm{H}(2 \mathrm{~b})$ & $6750(30)$ & $2700(70)$ & $4520(20)$ & $24(8)$ \\
$\mathrm{H}(3)$ & $5370(30)$ & $9210(80)$ & $3040(30)$ & $44(10)$ \\
$\mathrm{H}(1)$ & $8740(30)$ & $4260(80)$ & $4720(30)$ & $41(10)$ \\
\hline
\end{tabular}

Table 7

Torsion angles [deg]

\begin{tabular}{ll} 
Atoms & Dieder Angle \\
\hline $\mathrm{N}(3)-\mathrm{C}(2)-\mathrm{N}(2)-\mathrm{C}(1)$ & $-0.6(4)$ \\
$\mathrm{F}(2)-\mathrm{C}(2)-\mathrm{N}(2)-\mathrm{C}(1)$ & $177.1(2)$ \\
$\mathrm{N}(1)-\mathrm{C}(1)-\mathrm{N}(2)-\mathrm{C}(2)$ & $-0.5(4)$ \\
$\mathrm{F}(1)-\mathrm{C}(1)-\mathrm{N}(2)-\mathrm{C}(2)$ & $-178.6(2)$ \\
$\mathrm{N}(2)-\mathrm{C}(2)-\mathrm{N}(3)-\mathrm{C}(3)$ & $0.7(4)$ \\
$\mathrm{F}(2)-\mathrm{C}(2)-\mathrm{N}(3)-\mathrm{C}(3)$ & $-177.01(19)$ \\
$\mathrm{N}(4)-\mathrm{C}(3)-\mathrm{N}(3)-\mathrm{C}(2)$ & $179.2(2)$ \\
$\mathrm{N}(1)-\mathrm{C}(3)-\mathrm{N}(3)-\mathrm{C}(2)$ & $0.2(3)$ \\
$\mathrm{N}(2)-\mathrm{C}(1)-\mathrm{N}(1)-\mathrm{C}(3)$ & $1.2(4)$ \\
$\mathrm{F}(1)-\mathrm{C}(1)-\mathrm{N}(1)-\mathrm{C}(3)$ & $179.3(2)$ \\
$\mathrm{N}(4)-\mathrm{C}(3)-\mathrm{N}(1)-\mathrm{C}(1)$ & $180.0(2)$ \\
$\mathrm{N}(3)-\mathrm{C}(3)-\mathrm{N}(1)-\mathrm{C}(1)$ & $-1.0(3)$ \\
$\mathrm{N}(1)-\mathrm{C}(3)-\mathrm{N}(4)-\mathrm{C}(4)$ & $4.9(3)$ \\
$\mathrm{N}(3)-\mathrm{C}(3)-\mathrm{N}(4)-\mathrm{C}(4)$ & $-174.2(2)$ \\
$\mathrm{C}(5)-\mathrm{C}(4)-\mathrm{N}(4)-\mathrm{C}(3)$ & $75.6(3)$ \\
\hline
\end{tabular}

Table 8

Hydrogen bonds [A and deg.].

\begin{tabular}{lllll}
\hline $\mathrm{D}-\mathrm{H} . . . \mathrm{A}$ & $\mathrm{d}(\mathrm{D}-\mathrm{H})$ & $\mathrm{d}(\mathrm{H} \ldots \mathrm{A})$ & $\mathrm{d}(\mathrm{D} \ldots \mathrm{A})$ & $<(\mathrm{DHA})$
\end{tabular}

\section{CONCLUSIONS}

$\alpha$-Tocopherol turned out to be a versatile moiety for the development of new uracil derivatives as promising candidates for the introduction of lipophilic anchors into peptide nucleic acids (PNA) or peptides. Systems can be obtained containing one or two tocopherol units 
and carboxylic, amino or alkyne groups as tethering units. Sonogashira reaction turned out to be a useful tool in these approaches. An unusual phenomenon was found in 2-propargylamine-4,6difluorotriazine that exhibits two different chemical shifts for the two fluoro atoms.

\section{REFERENCES}

1. A. Kurz, A. Bunge, A. K. Windeck, M. Rost, W. Flasche, A. Arbuzova, D. Strohbach, S. Mueller, J. Liebscher, D. Huster and A. Herrmann, Angew. Chem. Int. Edit., 2006, 45, 4440-4444.

2. M. Loew, L. Kang, L. Dahne, R. Hendus-Altenburger, O. Kaczmarek, J. Liebscher, D. Huster, K. Ludwig, C. Bottcher, A. Herrmann and A. Arbuzova, Small, 2009, 5, 320-323.

3. D. Serien, C. Grimm, J. Liebscher, A. Herrmann and A. Arbuzova, New J. Chem, 2014, 38, 5181-5185.
4. M. Schade, A. Knoll, A. Vogel, O. Seitz, J. Liebscher, D. Huster, A. Herrmann and A. Arbuzova, J. Am. Chem. Soc., 2012, 134, 20490-20497.

5. M. Loew, R. Springer, S. Scolari, F. Altenbrunn, O. Seitz, J. Liebscher, D. Huster, A. Herrmann and A. Arbuzova, J. Am. Chem. Soc., 2010, 132, 16066-16072.

6. M. Loew, P. Pescador, M. Schade, J. Appelfeller, J. Liebscher, O. Seitz, D. Huster, A. Herrmann and A. Arbuzova, Eur. Biophys. J. Biophy., 2011, 40, 72-72.

7. M. G. Thomas, G. Maga, J. F. Mouscadet and N. M. Howarth, Nucleosides \& Nucleic Acids, 2007, 26, 1063 1066.

8. B. F. Marques and J. W. Schneider, Langmuir, 2005, 21, 2488-2494.

9. W. Flasche, C. Cismas, A. Herrmann and J. Liebscher, Synthesis, 2004, 2335-2341.

10. A. K. Feldman, B. Colasson and V. V. Fokin, Org. Lett. 2004, 6, 3897-3899.

11. L. Kosynkina, W. Wang and T. C. Liang, Tetrahedron Lett., 1994, 35, 5173-5176.

12. W. Flasche, C. Cismas, A. Herrmann and J. Liebscher, Synthesis, 2004, 2335-2341. 